\title{
Integrated time course omics analysis distinguishes immediate therapeutic response from acquired resistance
}

Genevieve Stein-O'Brien ${ }^{1,2+}$, Luciane T. Kagohara ${ }^{2 \dagger}$, Sijia Li ${ }^{2 \dagger}$, Manjusha Thakar ${ }^{2}$, Ruchira Ranaweera ${ }^{2,3}$, Hiroyuki Ozawa ${ }^{4}$, Haixia Cheng ${ }^{5}$, Michael Considine ${ }^{2}$, Sandra Schmitz ${ }^{6}$, Alexander V. Favorov ${ }^{2,7}$, Ludmila V. Danilova ${ }^{2,7}$, Joseph A. Califano ${ }^{8}$, Evgeny Izumchenko ${ }^{9}$, Daria A. Gaykalova ${ }^{9}$, Christine H. Chung ${ }^{2,3^{*}}$ and Elana J. Fertig ${ }^{2^{*}}$

\begin{abstract}
Background: Targeted therapies specifically act by blocking the activity of proteins that are encoded by genes critical for tumorigenesis. However, most cancers acquire resistance and long-term disease remission is rarely observed. Understanding the time course of molecular changes responsible for the development of acquired resistance could enable optimization of patients' treatment options. Clinically, acquired therapeutic resistance can only be studied at a single time point in resistant tumors.

Methods: To determine the dynamics of these molecular changes, we obtained high throughput omics data (RNA-sequencing and DNA methylation) weekly during the development of cetuximab resistance in a head and neck cancer in vitro model. The CoGAPS unsupervised algorithm was used to determine the dynamics of the molecular changes associated with resistance during the time course of resistance development.

Results: CoGAPS was used to quantify the evolving transcriptional and epigenetic changes. Applying a PatternMarker statistic to the results from CoGAPS enabled novel heatmap-based visualization of the dynamics in these time course omics data. We demonstrate that transcriptional changes result from immediate therapeutic response or resistance, whereas epigenetic alterations only occur with resistance. Integrated analysis demonstrates delayed onset of changes in DNA methylation relative to transcription, suggesting that resistance is stabilized epigenetically.

Conclusions: Genes with epigenetic alterations associated with resistance that have concordant expression changes are hypothesized to stabilize the resistant phenotype. These genes include FGFR1, which was associated with EGFR inhibitors resistance previously. Thus, integrated omics analysis distinguishes the timing of molecular drivers of resistance. This understanding of the time course progression of molecular changes in acquired resistance is important for the development of alternative treatment strategies that would introduce appropriate selection of new drugs to treat cancer before the resistant phenotype develops.
\end{abstract}

Keywords: Acquired resistance, Precision medicine, Data integration, Time course analysis, Genomics, Epigenetics

\footnotetext{
* Correspondence: Christine.Chung@moffitt.org; ejfertig@jhmi.edu

${ }^{\dagger}$ Genevieve Stein-O’Brien, Luciane T. Kagohara and Sijia Li contributed equally to this work.

2Department of Oncology, Sidney Kimmel Comprehensive Cancer Center,

Johns Hopkins University, Baltimore, MD, USA

Full list of author information is available at the end of the article
}

(c) The Author(s). 2018 Open Access This article is distributed under the terms of the Creative Commons Attribution 4.0 International License (http://creativecommons.org/licenses/by/4.0/), which permits unrestricted use, distribution, and reproduction in any medium, provided you give appropriate credit to the original author(s) and the source, provide a link to the Creative Commons license, and indicate if changes were made. The Creative Commons Public Domain Dedication waiver (http://creativecommons.org/publicdomain/zero/1.0/) applies to the data made available in this article, unless otherwise stated. 


\section{Background}

Recent advances to identification of gene regulation in cancer have enabled the selection of targeted therapies to inhibit specific regulators of oncogenic signaling pathways essential for tumor development and maintenance [1]. These therapies prolong survival but are not curative, since most patients develop acquired resistance within the first few years of treatment [2]. Although a wide variety of molecular alterations that confer resistance to the treatment have been described, the mechanisms and timing of their evolution are still poorly characterized $[3,4]$. As serial biopsies along the treatment period are impractical due to the invasiveness and high costs of the procedure, the molecular alterations associated with acquired resistance are only known when resistance has already developed and little is known about what changes occur at earlier or later time points during the targeted therapy. The lack of adequate in vitro and in vivo time course datasets makes it challenging to delineate the two predominant hypotheses for how therapeutic resistance develops: (1) the presence of small populations of resistant cells that will survive the treatment and repopulate the tumor; or (2) the development of de novo resistance mechanisms by the tumor cells $[4,5]$. Characterization of the dynamics of genomic alterations induced during acquired resistance can identify targetable oncogenic drivers and determine the best time point to introduce alternative therapeutic strategies to avoid resistance establishment [6].

Epidermal growth factor receptor (EGFR) inhibitors represent a common class of targeted therapeutics. Cetuximab, a monoclonal antibody against EGFR, is FDA approved for the treatment of metastatic colorectal cancer and head and neck squamous cell carcinoma (HNSCC) [7]. As with other targeted therapies, stable response is not observed for a long period and virtually all patients invariably develop acquired resistance [8]. Recent advances in the establishment of in vitro models of acquired cetuximab resistance [9] provide a unique opportunity to study the time course of genetic events resulting in acquired resistance. Cell lines chronically exposed to the targeted agent develop resistance and can be sequentially collected during the course of treatment to evaluate the progressive molecular changes. Previous studies to assess the mechanisms of acquired cetuximab resistance have been limited to comparing the genomic profile of the parental intrinsic sensitive cell line to stable clones with acquired resistance [9-11]. Therefore, these studies fail to capture the dynamics of acquired molecular alterations during the evolution of therapeutic resistance. The development of in vitro time course data to determine the molecular drivers of therapeutic resistance is crucial. These experimental systems have the further advantage that time course data can also be generated for untreated controls, enabling the distinction of the molecular mechanisms associated with acquired resistance from those that would occur due to the long-term culturing over the time period that resistance develops.

Along with novel time course datasets, inferring the specific and targetable signaling changes that drive therapeutic resistance also requires new bioinformatics pipelines to analyze and visualize these data. The bioinformatics pipelines must integrate genetic, epigenetic, and transcriptional changes from multiple-high throughput platforms to infer the complex gene regulatory mechanisms that are responsible for acquired resistance. Current supervised bioinformatics algorithms that find time course patterns in genomic data adjust linear models to correlate molecular profiles with known temporal patterns [12-15]. Many unknown variables such as culture conditions, immediate response to cetuximab, and adaptive changes may have confounding effects on known covariates of therapeutic response such as growth rates, colony size, or apoptosis rates. Unsupervised bioinformatics algorithms learn the dynamics directly from the high-throughput data, and therefore do not require a priori knowledge of the complex dynamics associated with therapeutic response. Some unsupervised algorithms [16-21] seek breaking points of coherent, regulatory relationships to infer the dynamics along pathways. Many of these algorithms trace individual phenotypes or individual genomics platforms. Their ability to determine drivers of gene expression associated with acquired resistance from time course data in multiple experimental conditions and multiple genomics data modalities is emerging [22]. Further extensions are needed to contrast the dynamics of signaling response to therapy to the dynamics of control conditions to distinguish the specific molecular processes that are unique to resistance. Matrix factorization algorithms are unsupervised and can distinguish the relative molecular changes in each experimental condition over time without requiring prior knowledge of gene regulation. We have found that Bayesian, non-negative matrix factorization algorithms such as Coordinate Gene Activity in Pattern Sets (CoGAPS) [23] can extend beyond clustering to robustly quantify the dynamics and infer the gene regulatory networks directly from the input time course data [24]. The CoGAPS error model can also be modified to enable datadriven inference in distinct molecular platforms for inference of epigenetic regulation of gene expression [25].

In this study, we developed a new bioinformatics analysis pipeline for integrated analysis of gene expression and DNA methylation changes that occur during the time course progression of resistance to targeted therapies using CoGAPS. Genes uniquely associated with these changes were selected using a PatternMarker statistic [26] to enable novel visualization of molecular 
alterations dynamics inferred with CoGAPS. In order to benchmark our new bioinformatics pipeline, we used an in vitro HNSCC cell line model to induce resistance and measure the molecular changes using high-throughput assays while the resistant phenotype developed. Gene expression and DNA methylation changes were screened weekly while acquired cetuximab resistance was induced in SCC25 cell line (intrinsic sensitive to cetuximab) and compared to the status of the untreated controls at the same culturing time point. CoGAPS [26] inferred specific patterns of expression and DNA methylation that are associated with the gradual establishment of acquired cetuximab resistance. The onset of methylation changes associated with resistance is temporally delayed relative to expression changes suggesting that epigenetic alterations stabilize the transcriptional changes relevant to the resistant phenotype. This analysis found anticorrelated changes between DNA methylation and gene expression in FGFR1 during acquired therapeutic resistance. Upregulation of FGFR1 has previously been associated as a mechanism of acquired cetuximab resistance in HNSCC [27-29]. The identification of a canonical marker of resistance to EGFR inhibitors in this present study corroborates the efficacy of our experimental model and analytical algorithm to identify mechanisms of resistance. To our knowledge, this is the first demonstration of the anti-correlation between FGFR1 methylation and expression suggestive of its epigenetic regulation in acquired resistance to cetuximab. Thus, this pipeline can identify mechanisms of gene regulation in acquired resistance from high-throughput, multi-platform time course data. The resulting bioinformatics pipeline is poised to infer the dynamics of acquired resistance from emerging time course data with other cancer types and therapeutics.

\section{Methods}

\section{Cell lines and materials}

SCC25 cells were purchased from American Type Culture Collection (ATCC). Cells were cultured in Dulbecco's Modified Eagle's medium and Ham's F12 medium supplemented with $400 \mathrm{ng} / \mathrm{mL}$ hydrocortisone and $10 \%$ fetal bovine serum and incubated at $37^{\circ} \mathrm{C}$ and $5 \%$ carbon dioxide. The parental cell line SCC25 and the late cetuximab and PBS generation 10 were authenticated using short tandem repeat (STR) analysis kit PowerPlex16HS (Promega, Madison, WI, USA) through the Johns Hopkins University Genetic Resources Core Facility. Cetuximab (Lilly, Indianapolis, IN, USA) was purchased from the Johns Hopkins Pharmacy.

\section{Induction of cetuximab resistance and time course sample collection}

The HNSCC cell line SCC25 (intrinsically sensitive to cetuximab) was treated with $100 \mathrm{nM}$ cetuximab every three days for 11 weeks (generations G1 to G11). On the eighth day, cells were harvested. Sixty thousand cells were replated for another week of treatment with cetuximab and the remaining cells were separately collected for: (1) RNA isolation (gene expression analysis); (2) DNA isolation (DNA methylation analysis); (3) proliferation assay; and (4) storage for future use. All steps were repeated for a total of 11 weeks. In parallel with the cetuximab treated cells, we generated controls that received the same correspondent volume of phosphate buffered saline (PBS). Cells were plated in several replicates each time at the same initial density. The replicates were then harvested and pooled to provide enough cells for genetic, epigenetic, and proliferation assays. To achieve adequate final cell confluence and number of cells for the experimental analysis of each generation, cetuximab- and PBS-treated cells were plated in different flask sizes. Cells treated with cetuximab were plated in multiple T75 $\left(75 \mathrm{~cm}^{2}\right)$ flasks $(60,000$ cells/flask $)$ that were combined on the eighth day. PBS-treated cells were plated in a single T175 $\left(175 \mathrm{~cm}^{2}\right)$ flask (60,000 cells). This design was selected considering the growth inhibition of the earliest cetuximab generations and to control confluence of the PBS controls at the collection time (Additional file 1: Figure S1).

\section{Cell proliferation and colony formation assays}

Cell proliferation events were measured using the Click-iT Plus EdU Flow Cytometry Assay Kit Alexa Fluor 488 Picolyl Azide (Life Technologies, Carlsbad, CA, USA) according to manufacturer's instructions. The cetuximab generations were considered resistant when the frequency of proliferating cells was higher than in the PBS control generations. Proliferation curves were generated using locally weighted polynomial regression (lowess) in R.

Anchorage-independent growth assay was used to further confirm the development of resistance. The parental SCC25 and the late G10 resistant cells were treated with different concentrations of cetuximab $10 \mathrm{nM}, 100 \mathrm{nM}$, and $1000 \mathrm{nM}$. Number of colonies was compared to the same cells treated with PBS. Colony formation assay in Matrigel (BD Biosciences, Franklin Lakes, NJ, USA) was performed as described previously [30].

Stable SCC25 cetuximab resistant single clones (CTXR clones) Resistance to cetuximab was induced in an independent passage of SCC25 cells. After resistance was confirmed, single cells were isolated and grown separately to generate the isogenic resistant single cell clones (CTXR). In total, 11 CTXR clones were maintained in culture without addition of cetuximab. With the exception of one clone (CTXR6), all CTXR clones presented substantial survival advantage compared to the parental SCC25, as reported by Cheng et al. [31]. Each of these clones was 
authenticated using STR analysis kit GenePrint 10 (Promega) through the JHU-GRCF, as previously published [31].

Proliferation assay was performed to confirm cetuximab resistance in the CTXR clones compared to the parental SCC25. A total of 1000 cells were seeded in 96-well plates in quadruplicate for each condition. PBS or cetuximab (10 nM, $100 \mathrm{nM}$ or $1000 \mathrm{nM}$ ) was added after 24 and $72 \mathrm{~h}$ and cells were maintained in culture for seven days. AlamarBlue reagent (Invitrogen, Carlsbad, CA, USA) at a $10 \%$ final concentration was incubated for $2 \mathrm{~h}$ and fluorescence was measured according to the manufacturer's recommendations (545 nm excitation, $590 \mathrm{~nm}$ emission). Resistance in the CTXR clones was confirmed when the proliferation rates were higher than in the PBS-treated SCC25 cells.

\section{RNA-sequencing (RNA-seq) and data normalization}

RNA isolation and sequencing were performed for the parental SCC25 cells (G0) and each of the cetuximab and PBS generations (G1 to G11) and the CTXR clones at the Johns Hopkins Medical Institutions (JHMI) Deep Sequencing \& Microarray Core Facility. RNA-seq was also performed for two additional technical replicates of parental SCC25 cell line to distinguish technical variability in the cell line from acquired resistance mechanisms. Total RNA was isolated from $1 \times 10^{6}$ cells using the AllPrep DNA/RNA Mini Kit (Qiagen, Hilden, Germany) following manufacturer's instructions. The RNA concentration was determined by the spectrophotometer Nanodrop (Thermo Fisher Scientific, Waltham, MA, USA) and quality was assessed using the 2100 Bioanalyzer (Agilent, Santa Clara, CA, USA) system. An RNA Integrity Number (RIN) of 7.0 was considered as the minimum to be used in the subsequent steps for RNA-seq. Library preparation was performed using the TrueSeq Stranded Total RNAseq Poly A1 Gold Kit (Illumina, San Diego, CA, USA), according to manufacturer's recommendations, followed by messenger RNA (mRNA) enrichment using poly(A) enrichment for ribosomal RNA (rRNA) removal. Sequencing was performed using the HiSeq platform (Illumina) for $2 \times 100$ bp sequencing. Reads were aligned to hg19 with MapSplice [32] and gene expression counts were quantified with RSEM [33]. Gene counts were upper-quartile normalized and log transformed for analysis following the RSEM v2 pipeline used to normalize TCGA RNA-seq data [34]. All RNA-seq data for the cell line mode in this study are available from GEO (GSE98812) as part of SuperSeries GSE98815.

\section{DNA methylation hybridization array and normalization}

Genome-wide DNA methylation analysis was performed on the same samples as RNA-seq using the Infinium
HumanMethylation450 BeadChip platform (Illumina) at the JHMI Sidney Kimmel Cancer Center Microarray Core Facility. Briefly, DNA quality was assessed using the PicoGreen DNA Kit (Life Technologies) and $400 \mathrm{ng}$ of genomic DNA was bisulfite converted using the EZ DNA Methylation Kit (Zymo Research, Irvine, CA, USA) following manufacturer's recommendations. A total volume of $4 \mu \mathrm{L}$ of bisulfite-converted DNA was denatured, neutralized, amplified, and fragmented according to the manufacturer's instructions. Finally, $12 \mu \mathrm{L}$ of each sample were hybridized to the array chip followed by primer-extension and staining steps. Chips were image-processed in the Illumina iScan system. Data from the resulting iDat files were normalized with funnorm implemented in the R/Bioconductor package minfi (version 1.16.1) [35]. Methylation status of each CpG site was computed from the signal intensity in the methylated probe $(M)$ and unmethylated probe $(U)$ as a $\beta$ value as follows:

$$
\beta=\frac{M}{M+U}
$$

Annotations of the $450 \mathrm{~K}$ probes to the human genome (hg19) were obtained from the R/Bioconductor package FDb.InfiniumMethylation.hg19 (version 2.2.0). Probes on sex chromosomes or annotated to single nucleotide polymorphisms were filtered from analysis. The CpG island probe located closest to the transcription start site was selected for each gene. Genes with CpG island probes $<200 \mathrm{bp}$ from the transcription start site were retained to limit analysis to $\mathrm{CpG}$ island promoter probes for each gene. Probes were said to be unmethylated for $\beta<0.1$ and methylated for $\beta>0.3$ based upon thresholds defined in TCGA analyses [34]. All DNA methylation data from this study are available from GEO (GSE98813) as part of SuperSeries GSE98815.

\section{Hierarchical clustering and CoGAPS analysis}

The following filtering criterion for genes from the profiling of the time course data from generations of cetuximab treated cells was used. Genes from RNA-seq data were selected if they had log fold change $>1$ between any two time points of the same condition and $<2$ between the replicate control samples at time zero (5940 genes). CpG island promoter probes for each gene were retained if the gene switched from unmethylated $(\beta<0.1)$ to methylated $(\beta>0.3)$ in any two samples of the time course $(1087$ genes). We used the union of the sets of genes retained from these filtering criteria on either data platform for analysis, leaving a total of 6445 genes in RNA-seq and 4703 in DNA methylation.

Hierarchical clustering analysis was performed with Pearson correlation dissimilarities between genes and 
samples on all retained genes. CoGAPS analysis was performed on both log transformed RNA-seq data and DNA methylation $\beta$ values, independently using the $\mathrm{R} /$ Bioconductor package CoGAPS [23] (version 2.9.2).

CoGAPS decomposes a matrix of data $\mathbf{D}$ according to the model

$$
D_{i, j} \sim N\left(\sum_{k=1}^{p} A_{i, k} P_{k, j}, \Sigma_{i, j}\right),
$$

where $N$ represents a univariate normal distribution, matrices $A$ and $P$ are learned from the data for a specified number of dimensions $P, \Sigma_{i, j}$ is an estimate of the standard deviation of each row and column of the data matrix $D$, and i represents each gene and $\mathrm{j}$ each sample. In this decomposition, each row of the pattern matrix $P$ quantifies the relative association of each sample with a continuous vector of relative gene expression changes in the corresponding column of $A$. That is each row of $P$ provides the relative magnitude across samples are called patterns and quantify the separation of distinct experimental conditions. These relative gene weights in the columns of $A$ represent the degree to which each gene is associated with an inferred pattern and are called metapathways. Together, these matrices provide a lowdimensional representation that reconstructs the signal of the input genomics data. A single gene may have non-zero magnitude in several distinct gene sets, representing the fact that a single gene can have distinct roles in different biological processes (such as immediate therapeutic response and acquired resistance). A recently developed PatternMarker statistic [26] selects the genes that are unique to each of the inferred patterns and therefore represent biomarkers unique to the corresponding biological process.

In the CoGAPS analysis of the data in this study, the standard deviation of the expression data was $10 \%$ of the signal with a minimum of 0.5 . The standard deviation of DNA methylation data under the assumption that $\beta$ values follow a beta distribution is

$$
\Sigma_{i, j}^{\beta}=\sqrt{\frac{\beta_{i, j}\left(1-\beta_{i, j}\right)}{M_{i, j}+U_{i, j}+1} .}
$$

CoGAPS was run for a range of 2-10 dimensions $P$ for expression and 2-5 for DNA methylation. Robustness analysis with ClutrFree [36] determined that the optimal number of dimensions $P$ for expression was 5 . DNA methylation was run in four parallel sets using GWCoGAPS [26]. In DNA methylation, the maximum number of patterns that modeled resistance mechanisms over and above technical variation in replicate samples of SCC25 was three. Gene sets representative of the meta-pathway were derived for each pattern using the PatternMarkers statistics [26]. Comparisons between DNA methylation and gene expression values for PatternMarkerGenes or from CoGAPS patterns and amplitudes were computed with Pearson correlation.

\section{Gene set analysis of cetuximab resistance signatures, the EGFR network, and pathways}

Gene set activity was estimated with the gene set statistic implemented in calcCoGAPSStat of the CoGAPS R/ Bioconductor package [23]. Analyses were performed on three gene sets: resistance signatures, gene targets of transcription factors in the EGFR network, and canonical pathways. Resistance signatures were defined based on previous literature. Specifically, in a previous study, CoGAPS learned a meta-pathway from gene expression data corresponding to overexpression of the $H R A S^{\text {Val12D }}$ in the $\mathrm{HaCaT}$ cell line model. That study associated the CoGAPS HaCaT-HRAS meta-pathway with gene expression changes in acquired cetuximab resistance in the HNSCC cell line UMSCC1 [23]. In the current study, we applied the PatternMarkers statistic [26] to the previously published CoGAPS analysis of these data to derive a gene set from this meta-pathway called HACAT HRAS_CETUXIMAB_RESISTANCE or HACAT_RESIS TANCE. In addition, we searched MSigDB [37] (version 5.2) for all gene sets associated with resistance to EGFR inhibition. In this search, we found the gene sets COL DREN_GEFITINIB_RESISTANCE_DN and COLDREN GEFITINIB_RESISTANCE_UP representing resistance to the EGFR inhibitor gefitinib in non-small-cell lung cancer (NSCLC) cell lines [38]. Gene sets of transcription factor targets were obtained from experimentally validated targets annotated in the TRANSFAC [39] professional database (version 2014.1). Canonical pathways were obtained from the C2 set of MSigDB [37] (version 6.1).

\section{Sources and analysis of additional in vitro and human tumor genomics data}

Genomics analyses of TCGA were performed on level 3 RNA-seq and DNA methylation data from the $243 \mathrm{HPV}$ negative HNSCC samples from the freeze set for publication [36]. DNA methylation data were analyzed for the same CpG island promoter probes obtained in the cell line studies. Pearson correlation coefficients were computed in $\mathrm{R}$ to associate different molecular profiles.

Additional analysis was performed on Affymetrix $\mathrm{Hu}$ man Genome U133 plus 2.0 GeneChip arrays for the SCC1/1CC8 isogenic cetuximab sensitive and resistant cell line pair described previously (GEO GSE21483 [30]). Additional gene expression data from SCC25 generated from the same platform in the same lab was also used for analysis, using fRMA for normalization [40] to control for batch effects as described previously [41]. 
Analysis was also performed on gene expression data measured with Illumina HumanHT-12 WG-DASL V4.0 R2 expression beadchip arrays on pre-treatment samples from patients subsequently treated with cetuximab from Bossi et al. [42], using expression normalization and progression-free survival groups as described in the study. Data were obtained from the GEO GSE65021 series matrix file.

DNA samples from eight human tumor surgical specimen post cetuximab treatment from the sample cohort in Schmitz et al. [43] were obtained for methylation profiling. Specifically, for each tumor one FFPE slide was stained with hematoxylin and eosin and tumor burden was evaluated. When the tumor content was $<50 \%$, the adjacent unstained FFPE slides were macrodissected in order to enrich the tumor burden. A double code was assigned to each sample by Biorepository. DNA was then extracted from two unstained slides using the QIAamp DNA FFPE Tissue kit. Briefly, slides were dipped into a xylene bath until paraffin was melted. Then slides were washed with ethanol $100 \%$ and tissue was harvested for extraction with QIAGEN affinity columns. The extracted DNA was quantified by NanoDrop spectrophotometer. DNA methylation was measured with the Illumina MethylationEPIC BeadChip (850K) array. Array data were normalized with the NOOB method [44] and converted to virtual $450 \mathrm{~K}$ arrays using the $\mathrm{R} /$ Bioconductor package minifi version 1.22 .1 and are available from GEO SuperSeries (GSE110995). Two samples had DNA content $<250 \mathrm{ng}$ and clustered separately from the remaining samples. These samples were filtered as low quality and excluded from the analysis, leaving six total tumor samples with DNA methylation data. Probes selected for the in vitro Illumina 450K DNA methylation data were used for subsequent analyses. Gene expression data from biopsy samples before cetuximab treatment and surgical samples after cetuximab treatment were obtained from the previous Schmitz et al. [43] study and normalized as described previously [41] and available from GEO SuperSeries (GSE110996).

We performed $\mathrm{t}$-tests and projections in $\mathrm{R}$ on the probe that had the highest standard deviation of expression values for each gene. CoGAPS signatures were also projected into these gene expression data using the methods described in Fertig et al. [23] with the ProjectR package version 0.99 .15 available from Github (https:// github.com/genesofeve/projectR). We also performed ttests in $\mathrm{R}$ to compare the long- (LPFS) and short-term progression-free survival (SPFS) groups based on the values obtained from this projection.

HNSCC samples and patient information collection were approved by the Independent Ethics Committee and the Belgian Health Authorities and conducted in accordance with the Declaration of Helsinki (October
2000). It was prospectively planned to perform translational research and patients gave their informed consent for repeated biopsies.

\section{Results}

Prolonged exposure to cetuximab induces resistance

Cetuximab resistance was induced by treating the SCC25 cells for a period of 11 weeks (CTX-G1 to G11). SCC25 cells treated with PBS were used as time-matched controls (PBS-G1 to -G11). Response to cetuximab was determined by comparing the proliferation rates between CTX and PBS generations. Proliferation of the PBS generations is stable throughout the 11 weeks (G1 to G11, Fig. 1a). Conversely, proliferation of the CTX generations progressively increases over each week (Fig. 1a). Relative to the untreated controls, the growth of the treated cells is initially (CTX-G1) inhibited until CTX-G3. Starting at CTX-G4, the absolute proliferation values are equal at this week, but the fit to the data across all time points suggests that the cells become resistant to the anti-proliferative effects of cetuximab and gain stable growth advantages compared to the untreated controls (CTX-G8 to -G11).

Comparison of proliferation rates between generations of CTX-treated cells relative to generations of cells treated with PBS enabled us to conclude that cell growth advantages arise from chronic cetuximab treatment and are associated with resistance rather than prolonged cell culturing. We mirrored the changes in proliferation rates with clinical responses seen in HNSCC tumors treated with cetuximab (Fig. 1b). The lower growth rates in CTX-G1 to -G3 may be an equivalent to the initial effects of the clinical treatment when the cancer cells are sensitive to cetuximab and reduction of the tumor size is observable. Even with gain in cell proliferation at CTXG3 and -G4, our model still corresponds to response to the treatment since the treated generations are not growing more than the controls (clinical stable tumor size). Finally, from CTX-G4 the higher proliferation even with cetuximab treatment is a representation of acquired resistance noticeable in the HNSCC patients as tumor recurrence or increase in tumor size.

Higher proliferation in treated than in untreated cells starts at CTX-G4 and we established this time point to call as the moment at which cetuximab resistance is stably acquired and all subsequent time points continue to develop acquired stable cetuximab resistance. To confirm this hypothesis, we evaluated the ability of the resistant CTX-G10 to anchorage-independent growth. Even under different concentrations of cetuximab, CTX-G10 presents enhanced anchorage-independent growth compared to the parental SCC25 (G0) (two-way ANOVA with multiple comparisons $p$ value $<0.01$ for each concentration, Additional file 1: Figure S2), 


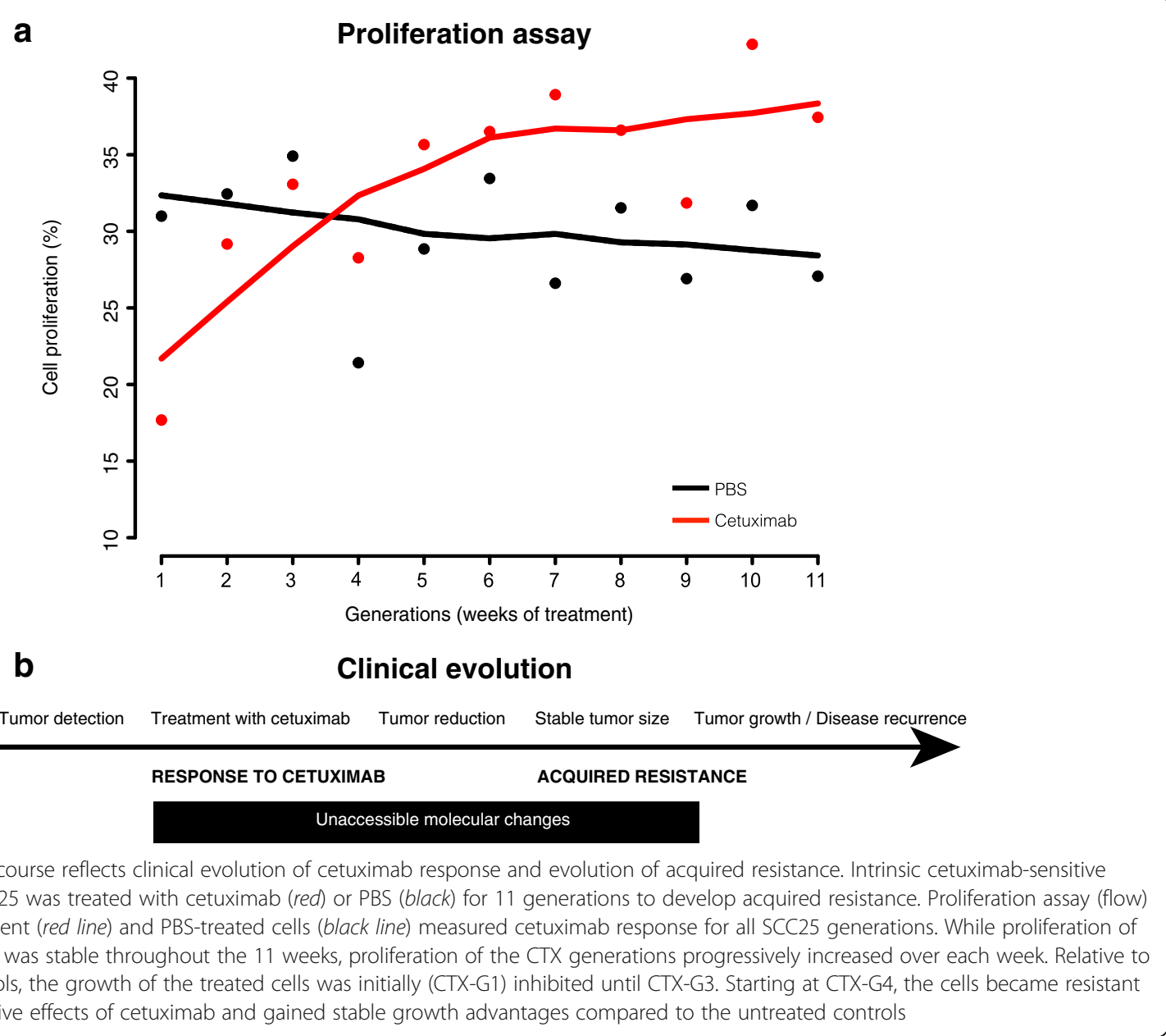

demonstrating the stabilization of cetuximab resistance in later generations.

\section{Treatment vs control gene expression changes governs clustering and immediate therapeutic response is confounded with changes from acquired resistance} RNA-seq data for the parental SCC25 cell line (G0) and from each generation of CTX- and PBS-treated cells were collected to characterize the gene expression changes occurring as cells acquired cetuximab resistance. Gene expression changes between treated (cetuximab) and untreated (PBS) cells and over generations of treated cells are apparent in time-ordered RNA-seq data (Fig. 2a). Additional clustering analysis of the samples accounting for the treatment time point (generations/ columns) (Additional file 1: Figure S3) distinguish three clusters of samples: those with cetuximab sensitivity (CTX-G1 to CTX-G3); those with early cetuximab resistance (CTX-G4 to CTX-G8); and those with late or stable cetuximab resistance (CTX-G9 to CTX-G11). The group of cetuximab sensitive samples corresponds to the time points at which the CTX generations present lower proliferation rates than the PBS controls (shown in Fig. 1a). The two groups of samples resistant to cetuximab are represented by a progressive increase in proliferation that is more significant than in the untreated controls (weeks 4 to 8 ) and by the stabilization in the proliferation rates (weeks 9 to 11), but still higher than in the PBS generations. The expression changes at the distinct time points during development of acquired resistance are shared among numerous genes. Although the clustering was able to separate cetuximab- from PBS-treated cells, it was not possible to discriminate the alterations related to an immediate therapeutic response (not relevant to the resistant phenotype) from resistance-specific gene expression changes.

Similar separation of the sensitive and resistant phases of cetuximab response is observed in clustering analysis of gene signatures previously described in HNSCC and NSCLC cell line models resistant to cetuximab or gefitinib (anti-EGFR small molecule), respectively [38, 41] (Additional file 1: Figure S4). For these genes, changes during early phases of resistance clusters for CTX-G4 to CTX-G6 as distinct from later generations CTX-G7 to 


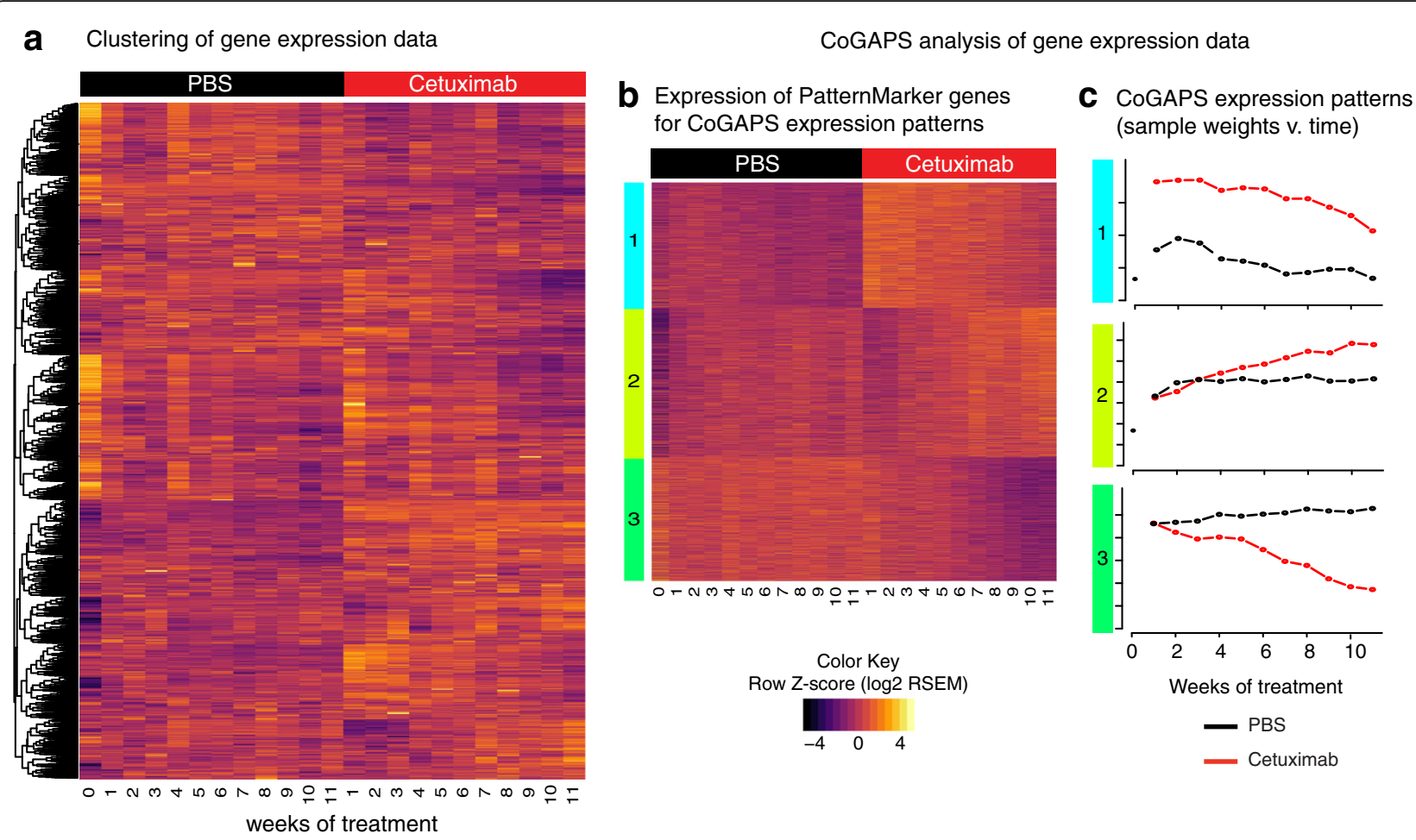

Fig. 2 CoGAPS analysis identifies signatures of resistance to EGFR inhibitors and separate resistant and control generations. a Heatmap of gene expression values in 11 generations of SCC25 cells treated with $100 \mathrm{nM}$ of cetuximab (red columns) to acquire resistance and with PBS as control (black columns). b Heatmap of gene expression values for PatternMarker genes identified with CoGAPS analysis of gene expression data from 11 generations of SCC25 cells treated with PBS as control (black columns) and with $100 \mathrm{nM}$ of cetuximab (red columns) to acquire resistance. Rows are colored according to which CoGAPS pattern the PatternMarker statistic assigned each gene and sorted by the PatternMarker statistic. c CoGAPS patterns inferred from gene expression data over generations of PBS control (black lines) or treatment with $100 \mathrm{nM}$ of cetuximab (red lines)

CTX-G11. Nevertheless, these signatures also cluster samples with gene expression changes at early phases (CTX-G1 to CTX-G3) as distinct from samples from PBS-treated generations. However, these analyses were insufficient to quantify the relative dynamics of genes associated with immediate response to therapy or subsequent acquired resistance.

\section{CoGAPS analysis of gene expression distinguishes patterns} of acquired resistance from immediate therapeutic response To define gene expression signatures for treatment effect and cetuximab resistance, we applied the CoGAPS [26] Bayesian matrix factorization algorithm to the time course gene expression data. CoGAPS decomposes the input data into two matrices: a pattern matrix with relative sample weights along rows and an amplitude matrix with relative gene weights along columns. Each row of the pattern matrix quantifies the extent of transcriptional changes within the genes in the corresponding column of the amplitude matrix and provides a low dimensional representation of the biological process in that data. In this analysis, we identified five CoGAPS patterns (Expression Patterns [EP]) (Additional file 1: Figure S5) in the time course gene expression dataset. A gene can have high amplitude in multiple patterns, modeling multiple regulation of genes but complicating visualization of the inferred patterns. A recently developed PatternMarker statistic defines genes that are uniquely associated with each of these patterns. Limiting the heatmap to these genes enables visualization of the dynamics of gene expression changes in our time course dataset (Additional file 1: Figure S5).

In this heatmap of CoGAPS PatternMarker genes, we observe that only three patterns (EP1, EP2, and EP3) distinguish the experimental conditions (cetuximab vs PBS) (top three patterns on Additional file 1: Figure S5). The other two patterns, EP4 and EP5, represent changes in gene expression from the parental cell lines and subsequent generations or an expression pattern that is constant and corresponds to signature of highly expressed genes (lower two patterns on Additional file 1: Figure S5), respectively. We note that highly expressed genes associated with EP5 may also have dynamic changes due to treatment and are filtered in the PatternMarker analysis of all patterns in Additional file 1: Figure S5. EP4 represents expression changes between treated cells and the parental cell line, which have a technical effect on gene expression. Notably, even the exclusion the flat 
pattern for highly expressed genes (EP5) still retains highly expressed genes in the signature. Retaining the technical pattern (EP4) in this calculation filters genes with expression changes from technical artifacts in the experimental conditions. The resulting set of PatternMarker genes for EP1-EP3 enable visualization of the expression changes dynamics that are associated with cetuximab response (Fig. 2b) and allow the definition of a gene signature associated with that response (Additional file 2: Table S1).

Similar to the separation seen with clustering (Additional file 1: Figure S5), the CoGAPS pattern EP1 distinguishes cetuximab from PBS at every generation (Fig. 2b and c, top). These genes present an immediate transcriptional upregulation in response to cetuximab treatment. Gene set analysis to determine the function of CoGAPS patterns was performed with an enrichment analysis on all gene weights in the amplitude matrix obtained from the CoGAPS analysis. By performing the analysis on gene weights and not only the PatternMarker genes, as shown in Fig. 2b, we account for multiple regulation of genes in pathways. Specifically, we performed gene set analysis on published resistance signatures [38, 41], transcription factors previously associated with the EGFR signaling network during cetuximab response in HNSCC $[39,41]$, and canonical pathways from MSigDB [37, 45] (Additional file 1: Figure S6; Additional file 3: Table S2). Gene set analysis confirms that published resistance signatures $[38,41]$ are significantly enriched in EP1 (Additional file 1: Figure S6; one-sided $p$ values of 0 . 002 and 0.003 for resistance gene sets COLDREN_GEFITINIB_RESISTANCE_DN and HACAT_HRAS_CETUX IMAB_RESISTANCE, respectively). However, the transcriptional changes in this pattern are not associated with acquired resistance to cetuximab and even decrease modestly as resistance developed. Further, enrichment by transcription factor AP-2alpha targets (TFAP2A; onesided $p$ value of 0.05 ) confirms previous work indicating that transcription by AP-2alpha is induced as an early feedback response to EGFR inhibition [39]. There are 84 significant canonical pathways from MSigDB, including notably pathways associated with the immune system, extracellular matrix, ERBB4 signaling, and VEGF signaling (Additional file 3: Table S2). Based upon these findings, we concluded that EP1 is associated with immediate response to cetuximab although it includes genes that are also associated with cetuximab resistance in previous studies.

The second CoGAPS expression pattern (EP2) quantifies divergence of the cetuximab treated cells from controls at generation CTX-G4 (Fig. 2b and c, middle) which is the time point that cetuximab treated cells present significant and stable growth advantage over PBS controls (Fig. 1a). Therefore, EP2 contains gene expression signatures associated consistently with the development of cetuximab resistance. Gene set statistics of transcription factor targets of EGFR on CoGAPS gene weights are significantly downregulated in this acquired resistance pattern (Additional file 1: Figure S6). One striking exception is c-Myc, which trends with acquired resistance ( $p$ value of 0.06), consistent with the role of this transcription factor in cellular growth. Resistance signature COLDREN_GEFITINIB_RESISTANCE_DN is significantly downregulated in EP2 ( $p$ value of 0.04 ). There are 32 statistically significant canonical pathways associated with this pattern, including notably telomerase, PI3K, and cell cycle pathways (Additional file 3: Table S2).

The third CoGAPS expression pattern (EP3) represents a gradual repression of gene expression with cetuximab treatment (Fig. $2 \mathrm{~b}$ and c, bottom). This expression pattern trends to significant enrichment in the COLDREN_GEFITINIB_RESISTANCE_DN resistance signature (Additional file 1: Figure S6, one-sided $p$ value 0. 12) and downregulated in the HACAT_HRAS_CETUXIMAB_RESISTANCE resistance signature (Additional file 1: Figure S6, one-sided $p$ value 0.09). This confirms that EP3 is associated with repression of gene expression during acquired cetuximab resistance. There are also 29 statistically significant canonical pathways associated with this pattern, including cell lineage, metabolic, WNT, and GSK3 pathways (Additional file 3: Table S2).

\section{Changes in DNA methylation inferred with CoGAPS are associated with resistance to cetuximab, but not the immediate response to treatment observed in gene expression}

To determine the timing of the methylation changes associated with acquired resistance, we also measured DNA methylation in each cetuximab generation of SCC25 cells and PBS controls (Fig. 3a). Application of the CoGAPS matrix factorization algorithm with the PatternMarker statistics to the methylation data reveals a total of three methylation patterns (MP) (Fig. 3bc; Additional file 2: Table S1): gradual increase of DNA methylation in controls (MP1, Fig. 3b middle); rapid demethylation in CTX generations starting at CTX-G4 (MP2, Fig. 3b bottom); and rapid increase in DNA methylation in CTX generations starting at CTX-G4 (MP3, Fig. 3b top). In contrast to the gene expression data, there is no immediate shift in DNA methylation resulting from cetuximab treatment. Gene set analysis was performed on canonical pathways from MSigDB (Additional file 4: Table S3) and found 26 statistically significant pathways for MP1, 29 for MP2, and 27 for MP3. In contrast to gene expression, the majority of canonical pathways is shared by the three methylation patterns and include notably the cytokine (PID-IL8- 


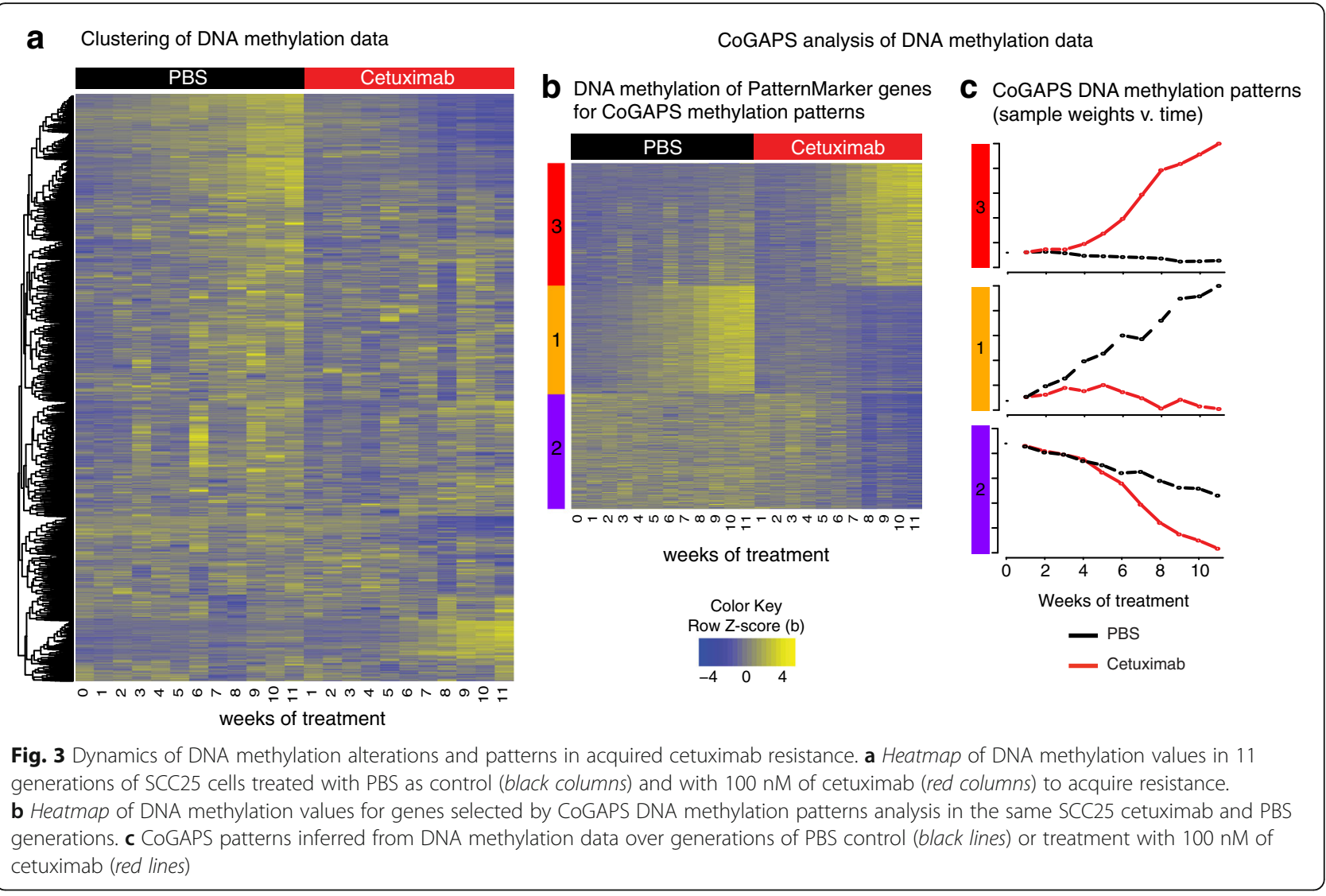

CXCR2 and IL8-CXCR1 pathways) and FGFR (Reactome Signaling by FGFR3 mutants) signaling pathways.

Comparing the CoGAPS patterns from gene expression and DNA methylation reveals strong anti-correlation between gene expression and DNA methylation in resistant patterns (Additional file 1: Figure S7A). We observed that the gene expression changes associated with acquired resistance occur gradually and are evident in early generations (Fig. 2c). The DNA methylation is consistent in cetuximab treatment and control PBS in DNA methylation patterns MP2 and MP3 during early generations; following which, there is a rapid accumulation in DNA methylation changes starting after generations CTX-G4 and CTX-G5 in both MP2 and MP3 (Fig. 3c), concurrent with the onset of the observed growth advantage over the PBS control (Fig. 1a).

While the patterns themselves are anti-correlated, the gene weights that define meta-pathways and are inferred in the amplitude matrix corresponding to each pattern with CoGAPS are not (Additional file 1: Figure S7B). We also observed little overlap between the PatternMarker genes from methylation patterns and gene expression. Changes in DNA methylation are delayed relative to those of gene expression in acquired cetuximab resistance as can be noted in Fig. 4, where direct comparison of the expression and methylation patterns previously shown (Figs. 2c and 3c, respectively) enable visualization of the time point when changes between cetuximab and PBS generations are significant in each pattern. These dynamics explain the discrepancy between the genes associated with each pattern and suggest that DNA methylation stabilizes the gene expression signatures crucial to the maintenance of acquired cetuximab resistance.

\section{Gene expression and methylation profile of SCC25 single-cell clones with acquired cetuximab resistance demonstrates cell heterogeneity}

The little overlap between the gene expression and DNA methylation PatternMarker genes and non-specific DNA methylation pathways may arise due to the development of different resistant sub-clones with specific gene signatures of acquired resistance in bulk data. In order to address this issue and to delineate whether our presumptive drivers resulted from clonal expansion of resistant cells or from the development of new epigenetic alterations to drive resistance, we measured DNA methylation and gene expression on a panel of 11 isogenic stable cetuximab-resistant clones (CTXR1 to CTXR11) derived from SCC25 cells in a previous study [31]. Despite being derived from the parental SCC25 cells after chronic exposure to cetuximab, the CTXR 


\section{Timing of separation in CTX and PBS}

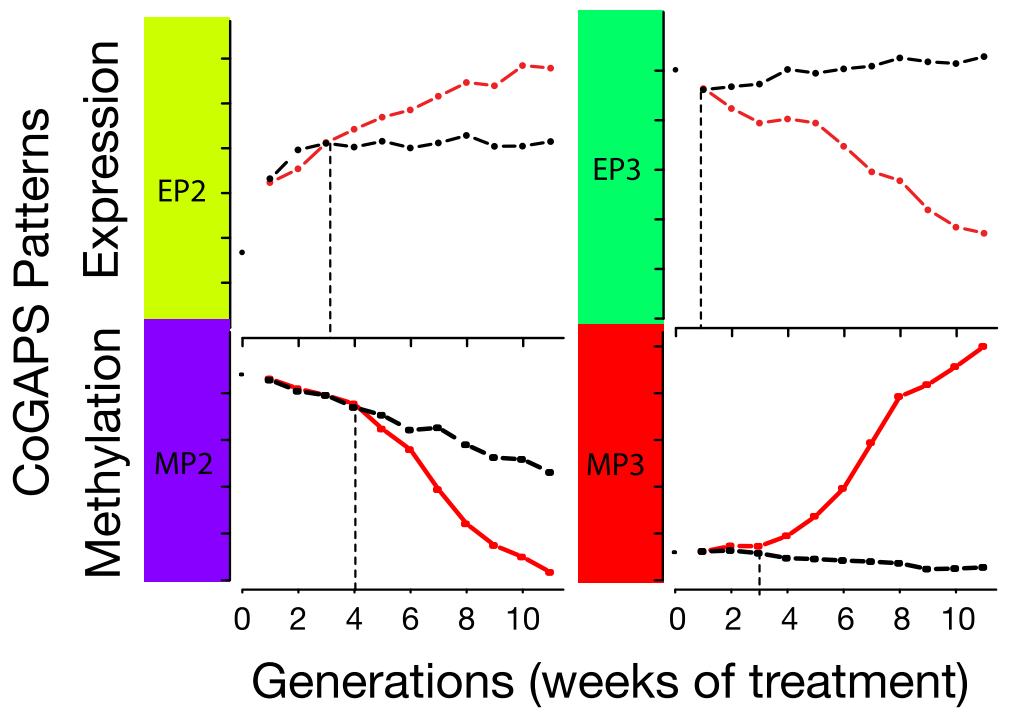

Fig. 4 DNA methylation and expression CoGAPS patterns demonstrate delayed onset of epigenetic changes in acquired resistance. CoGAPS patterns for gene expression (top) and DNA methylation (bottom) of patterns associated with acquired cetuximab resistance in SCC25 cetuximab generations (red) relative to PBS generations (black). Vertical dashed line represents time at which patterns for SCC25 generation separated from pattern for PBS generations. The timing of methylation changes distinguishing cetuximab-resistant generations was delayed in DNA methylation relative to that of gene expression

clones and the time course generations display widespread differences. We plot both expression and methylation profiles (Additional file 1: Figure S8 and Figure S9, respectively) among the DNA methylation PatternMarker genes that are anti-correlated with expression and cellular morphology (Additional file 1: Figure S10) for these clones. Significantly greater heterogeneity is observed among the CTXR clones in all these genomic and morphological data. Figure 5 demonstrates that higher heterogeneity among single cell clones is also observed in the epigenetically regulated PatternMarker genes from the CoGAPS analysis. These results suggest that different mechanisms of resistance may arise in the same HNSCC cell line as a result of intra-heterogeneity, resulting in the detection of a wide range of expression signatures with higher or lower correlation with the methylation profile depending on the size of each specific cell population.

FGFR1 overexpression and demethylation are associated with acquired cetuximab resistance in the time course and in stable cetuximab-resistant clones

To ascertain potential drivers of the stable cetuximabresistant phenotype induced by DNA methylation, we defined genes that are PatternMarkers [26] of the DNA methylation patterns associated with stable acquired cetuximab resistance (MP2 and MP3). We then applied correlation analysis to determine genes that were epigenetically regulated. Specifically, we performed correlation analysis between DNA methylation and gene expression for each of the DNA methylation PatternMarker genes (Fig. 5). This analysis identified FGFR1 as one of the genes with significant anti-correlation between expression and methylation, suggesting potential epigenetic regulation during cetuximab resistance acquisition. This finding is consistent with previous studies that associate differential expression of FGFR1 with resistance to EGFR inhibitors, including cetuximab, in HNSCC and other tumor types in vitro and in vivo [27, 46-48]. However, none of these studies demonstrate an association between FGFR1 upregulation and demethylation. Given the tight temporal regulation of the DNA methylation PatternMarkers with anti-correlated expression and the previous work on FGFR1, we hypothesize that this set of genes represents epigenetic drivers of acquired resistance.

We hypothesize that epigenetically regulated genes shared along the time course patterns and resistant single-cell clones might implicate common mechanisms acquired during evolution of the stable resistance phenotype. To test this assumption, we also performed correlation analysis for each of the epigenetically regulated genes in our resistant set (Fig. 5) in the resistant single cell clones and parental cell lines. Nine of the epigenetically regulated PatternMarker genes also have significantly anti-correlated gene expression and DNA methylation in the stable cetuximab-resistant clones. Of these, only FGFR1 is demethylated and re-expressed in a CTXR 


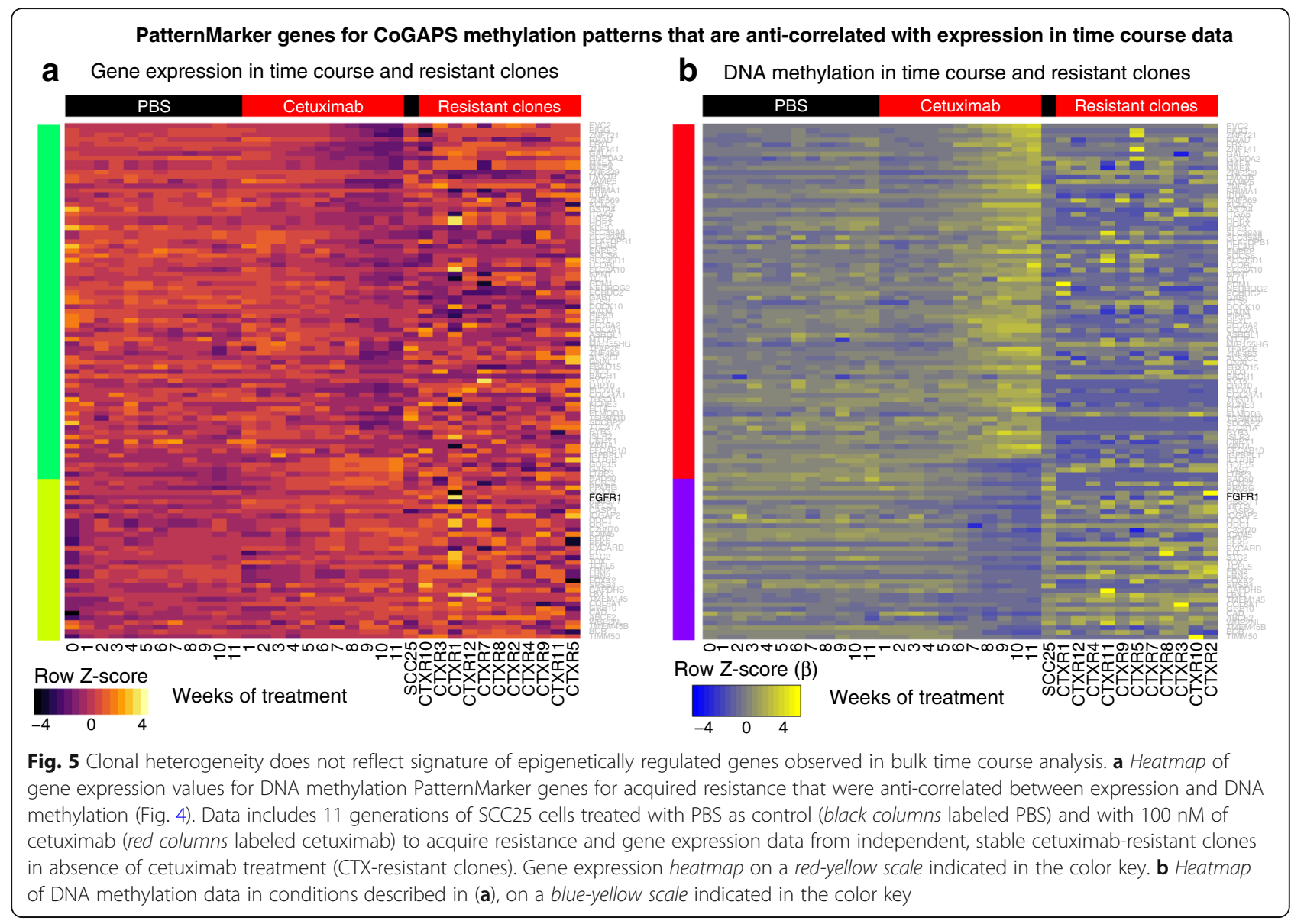

clone relative to the parental SCC25 cell line (Fig. 6a, remaining eight genes in Additional file 1: Figure S11). In this analysis, overexpression and de-methylation of FGFR1 expression occurs in only one of the resistant clones (CTXR10). QRT-PCR of FGFR1 gene expression in CTXR10 relative to the parental cell line demonstrated $\mathrm{a}>30$-fold change (Fig. 6b). Furthermore, in the resistant cell clone, increased levels of FGFR1 were associated with increased levels of phospho-FGFR1 and decrease in EGFR and phospho-EGFR as assessed by Western blot (Fig. 6c). This clone is one of the fastest growing under cetuximab treatment (Additional file 1: Figure S12). This observation suggests that the bulk data from the time course captured clonal outgrowth of a cetuximab-resistant clone with similar molecular features (FGFR1 demethylation) to CTXR10 and that clonal outgrowth is the dominant mechanism of resistance in our model.

\section{Observed FGFR1 dynamics in vitro recapitulates relationships from in vivo tumor genomics and acquired cetuximab resistance}

In order to confirm that the mechanisms we found with our in vitro approach are present in HNSCC samples pre and post cetuximab treatment, we further investigate the pattern of expression and methylation of FGFR1 and $E G F R$ in publicly available datasets. Using gene expression and DNA methylation data from The Cancer Genome Atlas (TCGA) for 243 HPV-negative HNSCC pre-treatment samples [36], we verified that the upregulation of EGFR and FGFR1 is not concomitant (Pearson correlation coefficient $=-0.06, p$ value $=0.33$, Fig. 7a). Additionally, the negative correlation of FGFR1 gene expression and DNA methylation status is statistically significant (Pearson correlation $\mathrm{r}$ of $-0.32, p$ value $<0.0001$, Fig. 7b), suggesting that FGFR1 transcription is associated with demethylation in some HPV-negative HNSCC tumors. Since there is no treatment information available for the TCGA dataset, we could not make assumptions related to cetuximab resistance and whether FGFR1 methylation is a consequence of the treatment. To assess this question, we collected new DNA methylation data for six HNSCC tumors after cetuximab treatment from a cohort of HNSCC tumor samples described previously [43]. All six samples have low DNA methylation values for FGFR1 ( $\beta$-values in the range of $0.04-0.08$, with a mean of 0.05 ), suggesting that the gene is unmethylated in these samples. While there was insufficient DNA to 
a

FGFR1 gene expression vs DNA methylation in resistant clones

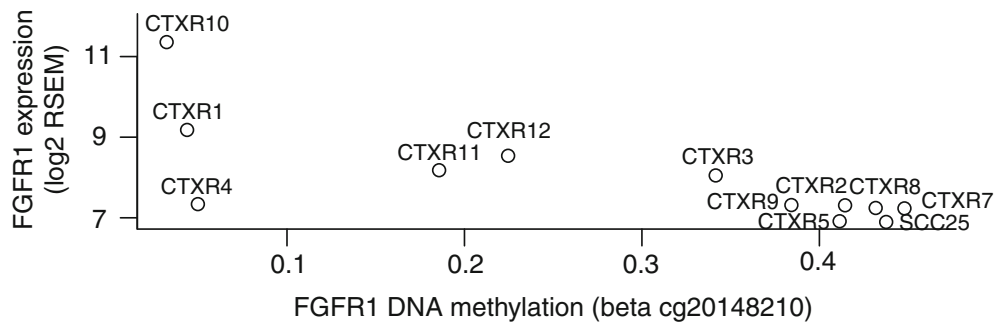

b

FGFR1 gene expression

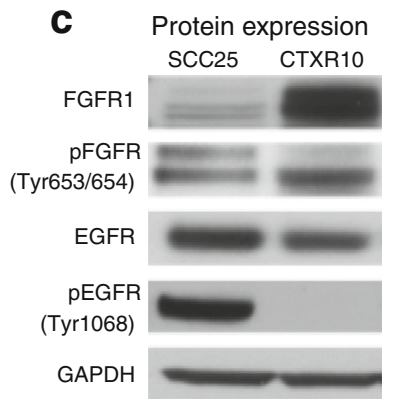

Fig. 6 Overexpression and de-methylation of FGFR1 in acquired cetuximab resistance is confirmed in stable SCC25-resistant clones. a Expression of FGFR1 relative to DNA methylation in stable cetuximab-resistant clones. b QRT-PCR of FGFR1 gene expression in CTXR10 relative to the parental cell line (> 30-fold change). c Western blot comparing FGFR1, phospho-FGFR1, EGFR, and phospho-EGFR in CTXR10 relative to the parental SCC25 cell line. In the resistant cell clone, increased levels of FGFR1 were associated with increased levels of phospho-FGFR1 and decrease in EGFR and phospho-EGFR

quantify DNA methylation before treatment in these patients, FGFR1 gene expression increases after treatment in four of the six tumor samples (Additional file 1: Figure S13). While this cohort is small, these data and TCGA suggest that FGFR1 methylation is potentially associated with its re-expression in HNSCC tumor samples in response to cetuximab treatment.

To determine whether FGFR1 is associated with cetuximab response, we used gene expression data from HNSCC patients before cetuximab treatment available from Bossi et al. [42]. After follow-up, the patients were separated in SPFS (median three months survival) or LPFS (median 19 months survival) according to time of recurrence or metastasis development. Using this dataset, we confirmed that EGFR expression in SPFS is significantly lower than in the LPSF group (Fig. 7c) (log fold change -1.0 , t-test $p$ value 0.0003 ). These data suggest that EGFR overexpression is associated with better response to cetuximab, consistent with the mechanism of action of the therapeutic. The opposite was observed for FGFR1, with overexpression in SPFS vs LPSF (Fig. 7d, log fold change 0.9 , t-test $p$ value 0.003 ). However, the Bossi et al. study [42] lacks DNA methylation data to assess whether FGFR1 was epigenetically regulated in these samples. Most patients with SPFS in this dataset also had intrinsic resistance to cetuximab, instead of acquired resistance studied in our in vitro model.
Nonetheless, these findings suggest that similar molecular mechanisms may contribute to both mechanisms (intrinsic and acquired) of cetuximab resistance in HNSCC.

\section{CoGAPS signatures of resistance and therapeutic response} replicate in an independent in vitro system and significantly stratified patient samples with longvs short-progression-free survival

To further illustrate that the results are reflective of HNSCC in a general fashion, we evaluated the behavior of the two additional cell lines and human tumors in the CoGAPS signatures using gene expression data available from previously published studies. The HNSCC cell lines SCC1 and 1CC8 were chosen as the cetuximab-resistant $1 \mathrm{CC} 8$ was generated from the cetuximab sensitive SCC1 in a similar protocol used to establish the single cell clones [30]. Data from SCC25 were also included as a reference. It is important to note that the treatment time for the SCC1 and 1CC8 pair is on the order of hours vs weeks, as used to generate the time course data. By projecting these data into the CoGAPS signatures, the relationship between the sensitive $\mathrm{SCC} 1$ and resistant $1 \mathrm{CC} 8$ recapitulates the relationship between PBS and CTX time course generations, respectively, in treatment driven signatures (Fig. 8a, b, e, f). Conversely, CoGAPS signatures related to culture specific conditions failed to produce meaningful differences between the lines (Fig. 

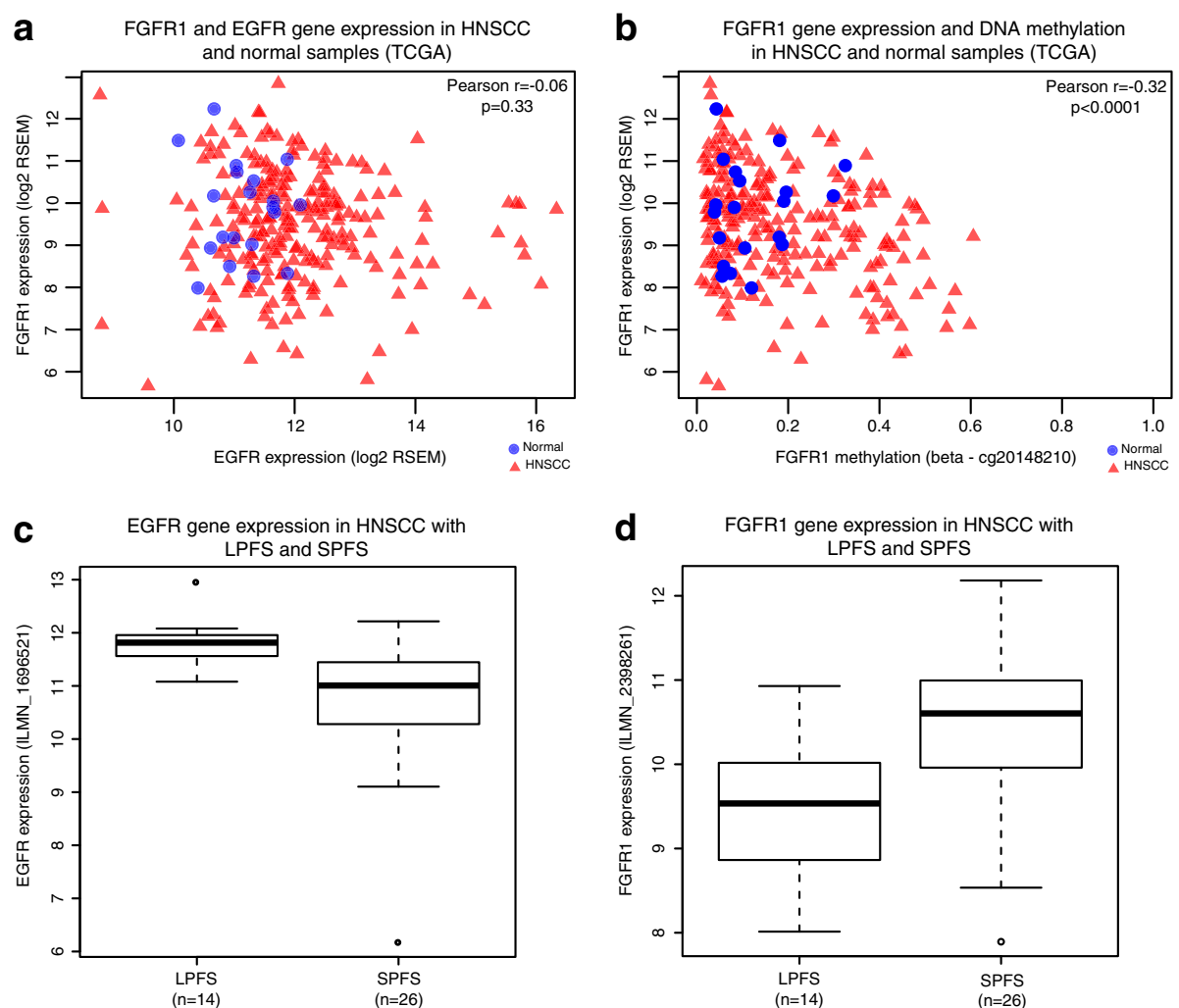

Fig. 7 FGFR1 gene expression and DNA methylation patterns were confirmed in independent HNSCC tumor sample datasets. a Scatter plot of gene expression for EGFR and FGFR1 in HPV-negative HNSCC samples from TCGA demonstrated that only a few HNSCC cases present increased levels of both genes and that there is no significant correlation between the expression of both genes concomitantly. $\mathbf{b}$ DNA methylation of FGFR1 was anti-correlated with expression in HPV-negative HNSCC TCGA samples, suggesting that upregulation of FGFR1 might be a result of promoter demethylation in primary tumors. c EGFR expression was significantly overexpressed in a group of HNSCC patients with LPFS relative to patients with SPFS in gene expression data from Bossi et al. d FGFR1 was significantly overexpressed in patients with SPFS relative to patients with LPFS in this same dataset. LPFS - long-progression-free survival; SPFS - short-progression-free survival

8c, d). Projections of the expression patterns from CoGAPS into the cell line gene expression data were also anti-correlated with projections of the methylation signatures in these same data. Gene expression data from HNSCC tumors from patients before their treatment with cetuximab described in Bossi et al. [42] were also analyzed. Projection into both the CoGAPS signatures of resistance and therapeutic response significantly stratified LPFS vs SPFS $\left(p\right.$ value $=5.2 \times 10^{-5}$ and $3.1 \times$ $10^{-3}$, respectively, (Fig. 8g, h). Conversely, projection in to the CoGAPS signature associated with culturing was not significant $(p$ value $=0.50$, Fig. $8 \mathrm{i})$.

\section{Discussion}

Analysis of course omics data enables separation of immediate treatment response and technical artifacts from molecular mechanisms of acquired therapeutic resistance

Although numerous short time course genomics studies of therapeutic response have been performed [49-51], this is the first time that genetic and epigenetic changes were measured for a prolonged exposure (11 weeks) to a targeted therapeutic agent. Using our novel robust time course integrated analysis approach, we characterized the molecular alterations during the development of acquired cetuximab resistance using a HNSCC in vitro model. Cell proliferation, gene expression, and DNA methylation high-throughput analysis were performed weekly in equivalent cultures (cetuximab and PBS control generations) as resistance developed. Over the course of 11 weeks, it was possible to compare treated (CTX) and untreated (PBS) cells grown under the same conditions. Applying robust bioinformatics algorithms, we discriminated changes associated with acquired resistance from those related to adaptive response to the cell culturing process and treatment. The SCC25 cell line model was chosen since this is one of the only two HNSCC cell lines previously used to generate isogenic cetuximab-resistant cell lines [10]. However, this is the first study to our knowledge to enable characterization of the transcriptional and epigenetic dynamics at the early phases of therapeutic resistance. These phases 


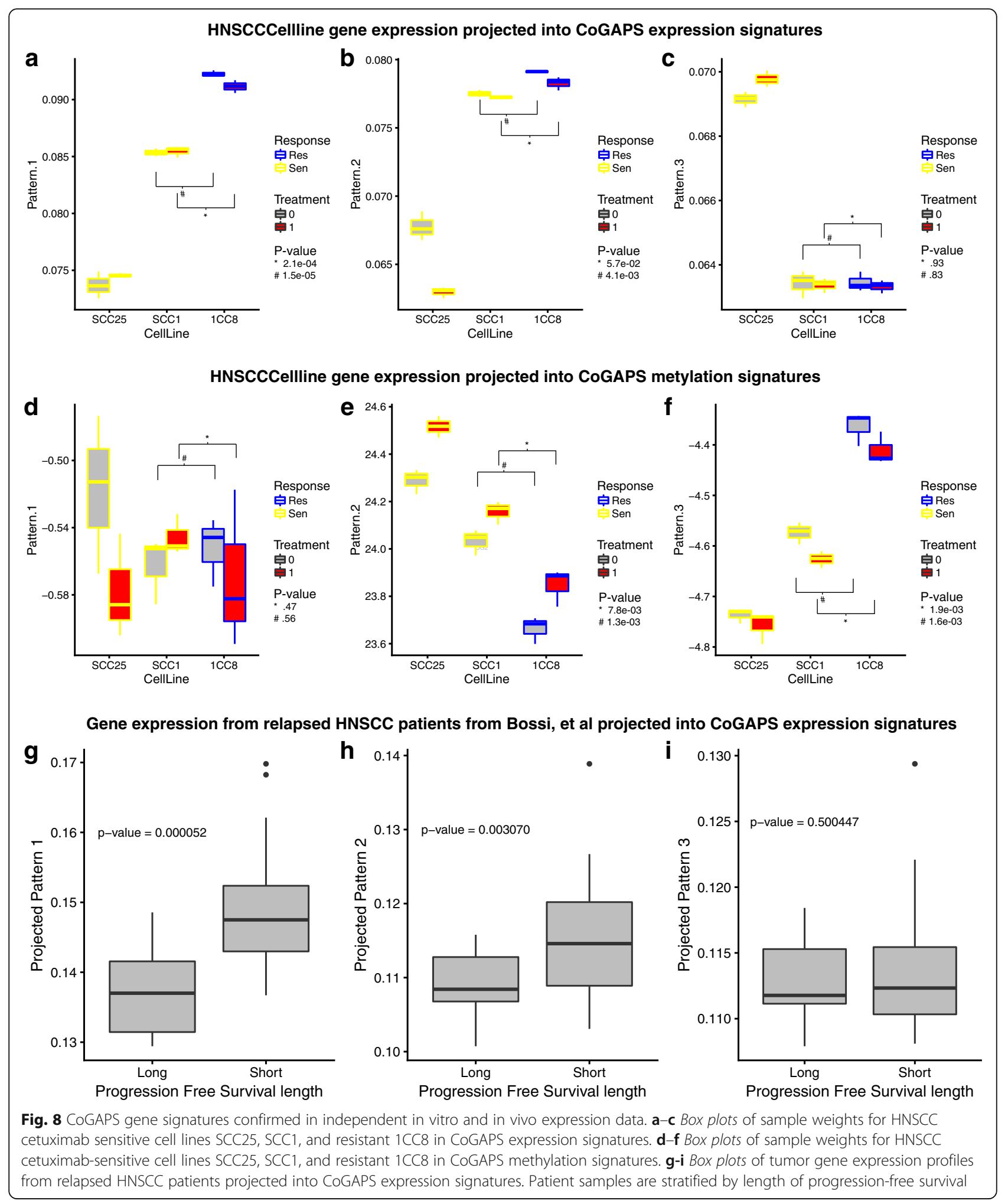


cannot be measured in patients due to the complexity of early detection of resistance and obtaining repeated biopsy samples.

\section{Unsupervised time course analysis with CoGAPS quantifies, visualizes, and enables functional analysis of the dynamics of acquired therapeutic resistance across omics data modalities}

Determining the dynamics of the molecular alterations responsible for resistance requires integrated, time course bioinformatics analysis to quantify these alterations. Based upon previous performance of Bayesian, non-negative matrix factorization algorithms in inferring dynamic regulatory networks for targeted therapeutics $[49,50]$, we selected CoGAPS [23] for analysis of gene expression data from our time course experiment. CoGAPS have already proven highly effective in relating gene expression changes to patterns related to EGFR inhibition [41], perturbation of nodes in the EGFR network [39], and time course dynamics of targeted therapeutics. In this dataset, CoGAPS analysis of gene expression data from cetuximab-resistant clones distinguished the patterns for immediate gene expression changes and patterns for long-term changes associated with acquired resistance. Gene expression signatures for resistance to EGFR inhibitors in two additional cell lines (one HNSCC and one NSCLC) from previous studies $[38,41]$ were significantly enriched in both types of CoGAPS patterns. Since these previous resistance signatures were learned from case-control studies without multiple time point measurements, we concluded that our time course data are instrumental in discriminating the signatures of immediate therapeutic response from signatures of acquired resistance.

In spite of the complexities of the data integration, the weight of each sample in patterns inferred by CoGAPS reflects the dynamics of the process in each data modality. These patterns are learned completely unsupervised from the data and do not require any gene selection or comparison between time points relative to any reference control. The CoGAPS analysis of the time course data demonstrates that applying matrix factorization algorithms for genomics can reconstruct signals associated with phenotypes from time course, omics data. The genes associated with CoGAPS patterns had weights that were non-zero in multiple patterns. The PatternMarker statistic [26] enable further selection of the genes that are uniquely associated with each pattern. Creating a heatmap of the genomics profiles for these genes enabled novel, heatmap-based visualization of the temporal dynamics in the omics data. CoGAPS analysis of gene expression data contains a flat pattern (EP5), which includes all highly expressed genes. These genes may also change in association with the experimental conditions, albeit to a lesser degree than lowly expressed genes. Because the PatternMarker statistics includes genes that are uniquely associated with each inferred biological process, these highly expressed genes would be filtered from associations with the dynamic conditions. To include these genes in the signatures defined in this study, EP5 was filtered from the calculation of the PatternMarker statistic. Such filtering process is not required for heatmap-based visualization and filtering of flat patterns is recommend when defining gene signatures containing sets of genes that are most strongly associated with dynamic changes. Patterns that reflect technical artifacts in the data, such as EP4, should be retained in the PatternMarker analysis to limit the signatures associated with inferred processes to retain only biologically relevant genes. We note that these PatternMarker statistic are similar to the D-scores proposed in Zhu et al. [52] and that application of this statistic may require similar filtering to retain highly expressed genes. In the case of DNA methylation, these PatternMarker genes also include genes representing driver alterations in resistance. The DNA methylation data did not require filtering when applying the PatternMarker statistic since no flat pattern was detected. However, transcriptional regulation by epigenetic alterations or in pathways involves simultaneous co-regulation of multiple genes. This coregulation is reflected in the reuse of genes in CoGAPS gene weights associated with each pattern. Therefore, estimates of pathway dynamics from transcriptional data require accounting for all genes with gene set enrichment statistics instead of the PatternMarker statistic. Thus, we hypothesize that the PatternMarker statistic is robust for visualization and biomarker identification. On the other hand, gene set enrichment of the CoGAPS gene weights corresponding to each pattern and stored in the amplitude matrix are essential for characterization of functional alterations in pathways.

\section{Integrated genomics analysis of time course data demonstrates that DNA methylation changes follow transcriptional changes, leading to the model where methylation stabilizes the resistance phenotype}

Collecting treated and untreated cells to obtain paired measurements of methylation and gene expression enabled us to evaluate whether changes in DNA methylation impact gene expression. Including a PBS control at every time point also enabled the discrimination of the changes that result from an adaptive response to therapy from changes that result from maintaining cells in culture. CoGAPS analysis of DNA methylation data denotes only changes associated with acquired resistance, in contrast to the immediate expression changes observed with cetuximab treatment. Thus, while therapeutic response can drive massive changes in gene expression, only the 
subset of expression changes associated with the development of resistance have corresponding epigenetic signatures, suggesting that the methylation landscape is important for the development of acquired resistance. The CoGAPS patterns in gene expression that are associated with acquired cetuximab resistance gradually change over the time course (EP1 and EP2). On the other hand, the CoGAPS patterns for DNA methylation changes have a sharp transition at the generation at which resistance is acquired (CTX-G4). These patterns (MP2 and MP3) reflect a delayed but more rapid change in DNA methylation. The time delays between alterations in DNA methylation and gene expression pose a further computational challenge for integrated, time course genomics analyses. The vast majority of integrated analysis algorithms assume one-to-one mapping of genes in different data platforms or seek common patterns or latent variables across them [53]. Such approaches would fail to capture the early changes from cetuximab treatment that impact only gene expression; time delays between DNA methylation and gene expression patterns and different gene usage in each pattern. It is essential to develop new integrated algorithms to simultaneously distinguish both patterns that are shared across data types and that are unique to each platform. For time course data analysis, these algorithms must also model regulatory relationships that may give rise to timing delays, such as epigenetic silencing of gene expression. However, as we observed with the unanticipated changes in DNA methylation following and not preceding gene expression, they must also consider delays resulting from larger phenotypic changes such as the stability of the therapeutic resistant phenotype.

The relative timing of change in DNA methylation and gene expression is consistent with previous observations that gene expression changes precede DNA methylation alterations in genes critical for cancer progression. $P 16^{I N K 4 A}$ and GSTP1 are tumor suppressor genes for which transcription silencing was found to occur prior to DNA hypermethylation and chromatin changes. The temporal delay observed between expression and methylation patterns in our time course provides transcriptome-wide evidence of this phenomenon. Specifically, that epigenetic changes are necessary to stabilize gene expression aberrant profile and will be followed by modification into a silenced methylation state, resulting in tumor progression $[54,55]$. Our integrated RNA-seq and DNA methylation analysis corroborates the fact that gene expression changes occur earlier to epigenetic alterations and suggests that DNA methylation is essential to maintain the changes in gene expression in this acquired cetuximab resistance model. Additional time course data tracing other in vitro and in vivo models of HNSCC are essential to generalize the relative timing of molecular changes, and thus mechanisms of gene regulation, associated with acquired therapeutic resistance. Future investigation into the chromatin remodeling mechanisms will also test whether chromatin alterations follow the changes in expression and occur in combination with altered methylation patterns to drive epigenetic regulation of resistance.

\section{Time course data are essential to distinguish clonal outgrowth from transcriptional rewiring that give rise to stable acquired resistance to therapy}

Besides the immediate changes in gene expression followed by the gradual methylation switch, it is also interesting to note these effects in the proliferation rates during the 11 weeks of treatment. Initially, the proliferation of the population of cetuximab-sensitive cells is slower when compared to the untreated controls, reflecting therapy effectiveness. Early and progressively, the cells develop molecular changes to overcome the EGFR blockade. However, this process starts in just a small number of clones and the increase in proliferation is still not enough to surpass the growth rate of the untreated cells. As soon as the population of resistant cells is larger than the number of sensitive cells, the proliferation rate is now higher than in the untreated controls. At some point, we observe the stabilization of the proliferation rates in the cetuximab-treated cells, probably due to the fact the culture is now dominated by the population of resistant clones. Although stable, the proliferation rate of these resistant clones is significantly higher than that of the untreated cells. This increased proliferation rate is consistent with the rapid increase in tumor volume observed clinically once patients develop resistance to therapy. Tracing the increase in the population of resistant cells and their proliferation rates in vivo requires novel techniques to biopsy or image tumors at intermediate time points of treatment.

In a recent study, gene expression changes were associated with a transient resistant phenotype present in melanoma cell lines before vemurafenib administration [56]. Once the melanoma cells were exposed to the drug, additional changes in gene expression are detected and are later accompanied by changes in chromatin structure [56]. These findings, together with our time course observations, suggest that in the heterogeneous tumor environment the existence of some cells expressing specific marker genes can trigger cellular reprogramming as soon as the targeted therapy is initiated. Upon drug administration, the number of genes with aberrant expression increases and is followed by other epigenetic and genetic changes that will shift the transient resistant state into a stable phenotype. This finding on acquired resistance development could dramatically change the course of treatment with targeted therapeutic agents. 
The precise characterization of resistant gene signatures and their timing are crucial to determine the correct point during the patients' clinical evolution to introduce alternative therapeutic strategies. This way, secondary interventions would start before the stable resistant phenotype is spread among the tumor cells resulting in prolonged disease control and substantial increase in overall survival.

\section{DNA methylation changes in FGFR1 are associated with changes in FGFR signaling}

Among the genes we identified with the canonical relationship between expression and methylation, FGFR1 present with increased gene expression accompanied by loss of CpG methylation. FGFR1 is a receptor tyrosine kinase that regulates downstream pathways, such as PI3K/AKT, and RAS/MAPK, which are also regulated by EGFR [57]. Its overexpression has been previously associated with resistance to EGFR inhibitors in other cancer types including HNSCC [27-29]. To our knowledge this is the first study showing that epigenetic alterations are associated with changes in FGFR1 expression in HNSCC during the development acquired cetuximab resistance. FGFR1 upregulation combined with promoter hypomethylation was previously described in rhabdomyosarcomas [58]. Other studies described that FGFR1 increased levels is a common feature in different tumor types, such as glioblastoma [59] and cancers of the breast [60], lung [61], prostate [62], bladder [63], ovarian [64], colorectal [27], and HNSCC $[29,65,66]$. FGFR1 is involved in resistance mechanisms against EGFR inhibitors [27, 46-48], such as cetuximab and gefitinib. Together, the TCGA and Bossi et al. dataset analyses corroborate our findings that FGFR1 gene expression is regulated by epigenetic changes in HNSCC. Altogether, the epigenetic alteration of FGFR1 represents a candidate biomarker of resistance to cetuximab and further studies are critical to identify combination therapies for HNSCC patients that develop acquired cetuximab resistance.

The increased levels of FGFRs and FGFs are believed to play a role in an autocrine mechanism in HNSCC and NSCLC cell lines with intrinsic resistance to the EGFR inhibitor, gefitinib. Using publicly available gene expression microarray datasets, Marshall et al. [47] and Marek et al. [46] verified concomitant increased levels of FGFRs and their specific FGFs ligands. Particularly, FGFR1 and FGF2 upregulation was observed in the same resistant cell lines and hypothesized to be the mechanism behind resistance. This was corroborated by functional experiments showing that cells treated with pan-FGFR inhibitor were less prone to anchorageindependent growth. Also, FGF2 silencing or FGFR1 inhibition resulted in phospho-ERK decreased expression that was restored when FGF2 was added to the culture, suggesting that an autocrine FGF-FGFR pathway is one of the mechanisms of resistance to gefitinib. However, the cell lines evaluated in both studies were intrinsically resistant to gefitinib. In our model, we induced resistance to cetuximab and observed FGFR1 gain of expression and significant anti-correlation with the DNA methylation. We additionally evaluated the expression of other FGFRs and FGFs that were identified by the PatternMarker statistic. Although it is not found as a PatternMarker in our analysis pipeline, FGF2 is upregulated in the cetuximab generations when compared to the PBS generations as observed with FGFR1 (Additional file 1: Figure S14). Thus, our data corroborates and extends this previous evidence from intrinsically resistant lines that one of the mechanisms driving resistance to EGFR inhibition is the FGF-FGFR autocrine pathway. This observation adds another evidence that the computational approach used in this study is robust once it is capable of identifying mechanisms previously described in other models resistant to EGFR blockade.

Our previously developed bioinformatics algorithms for the identification of gene expression and epigenetic patterns progression over time proved to be consistent, since they also detected canonical changes found to be driving this mechanism among innumerous new potential candidates for acquired resistance. The integrated computational analysis was possible due to an experimental approach developed to account for molecular changes due to adaptive responses to the culturing system and the immediate addition of cetuximab. Here, we present a novel integrated analysis protocol to evaluate molecular changes measured by different high throughput techniques over a prolonged time of treatment with an FDA-approved targeted therapeutic agent. The lack of in vivo experimentation to validate our findings was compensated by the analysis of two public datasets of HNSCC, showing that our in vitro findings were also present in patients' samples. Our findings, together with Marshall et al. [47] and Marek et al. [46], are a strong evidence that FGFR1 plays a crucial role in a significant proportion of cases that are resistant to cetuximab or gefitinib. The translational implications are notable since FGFR1 inhibition can be used in combination with EGFR blockade to retard acquired resistance or overcome intrinsic resistance. It is important to mention that FGFR inhibitors are being currently evaluated by clinical trials and could soon become a potential new therapeutic option for many cancer patients [57]. Future work evaluating how these combinations impact the timing of acquired resistance are essential to determine the molecular mechanisms that shift 
dominant signaling pathways in cancer and thereby drive resistance.

\section{CoGAPS gene signatures from the SCC25 time course model are associated with molecular changes in additional in vitro models and human tumor data}

The main limitation of the current study was the use of a single cell line model. SCC25 is intrinsically sensitive to cetuximab and from this single cell line model, we generated two groups of samples (CTX and PBS generations) over the course of 11 weeks. High-throughput measurements and analysis were performed for a total of 22 samples. The collection of multiple data points in the analysis had to be accounted for when determining the number of cell lines to be included in the study. We nonetheless compared our data to gene signatures from the other isogenic HNSCC resistant model 1CC8 [10], an independent resistant model to an EGFR inhibitor in NSCLC [38], and human tumor data from HNSCC patients before cetuximab treatment [42]. Besides the number of samples, we also had to take into consideration the potential batch and technical effects of broad cross-platform profiling. Nevertheless, the analysis of pretreatment HNSCC patient samples from TCGA [36] and another study [42] confirmed that our finding that FGFR1 is upregulated and demethylated in HNSCC and associated with acquired resistance to cetuximab is also a mechanism involved in intrinsic resistance to the targeted therapy.

\section{Transcriptional heterogeneity is critical in acquired} therapeutic resistance, and future time course single cell data will pinpoint precise molecular predictors of therapeutic resistance

The in vitro protocol for time course sampling developed in this study has the additional advantage of aggregating potentially heterogeneous mechanisms of resistance increasing the signal of changes in any cetuximab-resistant sub-clone. For example, we observed demethylation and overexpression of FGFR1 in the pooled cells, but only a single stable clone generated from the same SCC25 cell line in a previous study (CTXR10) had upregulation of FGFR1 [31]. This finding suggests that tumor heterogeneity also plays a role in acquired resistance to targeted therapies and enables different pathways to be used to bypass the silenced target within the same tumor. Heterogeneity of SCC25 cetuximab-resistant clones has been observed previously [31]. Recent single cell RNA-seq data of SCC25 has shown that there is considerable transcriptional heterogeneity in this cell line before treatment [67]. Other cancer therapies are influenced by heterogeneity and outgrowth of resistant clones, as was observed in single cell clones isolated from the HNSCC cell line FaDu when treated with cisplatin [68]. These data and the intrinsic sensitivity of SCC25 to EGFR inhibition suggests that therapeutic resistance results from random selection of a pre-existing resistant clone. The heterogeneity in methylation profiles reflected the complexity of the resistance mechanisms that can arise from combination therapies in heterogeneous tumors. Future work extending these protocols to in vivo models is essential to determine the role of the microenvironment in inducing therapeutic resistance. Developing in vivo models with acquired therapeutic resistance presents numerous technical challenges that must first be addressed before such time course sampling is possible [9]. Pinpointing precise molecular predictors of therapeutic resistance will facilitate the identification of unprecedented biomarkers and reveal the mechanisms by which to overcome acquired therapeutic resistance to most therapies used to treat cancer.

\section{Conclusions}

By developing a novel bioinformatics pipeline for integrated time course analysis, we measured the changes in gene expression and DNA methylation during the progression from an intrinsic cetuximab responsive state to the acquired resistant phenotype using an in vitro HNSCC cell line model. Specifically, this pipeline includes: (1) CoGAPS analysis of each platform independently; (2) gene selection with the PatternMarker statistic for visualization and CoGAPS gene set analysis of the CoGAPS gene profiles for pathway analysis; (3) comparisons of patterns to known phenotypes infer their relative timing; (4) anti-correlation between DNA methylation patterns and gene expression to infer epigenetically regulated genes; and (5) evaluation of PatternMarker genes and projection of the CoGAPS gene profiles to learn relevance of inferred gene signatures in new datasets. This pipeline revealed massive changes in gene expression and identified and discriminated the different patterns associated with resistance or cell culturing conditions. This analysis demonstrates that compressed sensing matrix factorization algorithms can identify gene signatures associated with the dynamics of phenotypic changes from genomics data collected over the time course. In this case, the gene expression patterns relevant to resistance were later followed by epigenetic alterations. Our main conclusion is that using our bioinformatics approach we are able to determine that the resistant phenotype is driven by gene expression changes that would confer the cancer cells adaptive advantages to the treatment with cetuximab. Finally, the integrated analysis show that the stability of the resistant state is dependent on epigenetic changes that will make these new gene signatures heritable to expand the phenotype to the daughter cells. The 
bioinformatics pipeline we developed is also significant to clinical practice, since it pointed the time course of molecular changes associated with acquired cetuximab resistance and suggests that the resistant phenotype can be reversed if alternative interventions are introduced before epigenetic alterations to the genes driving acquired resistance. Moreover, the computational approach we describe here can be applied to time course studies using other tumor type models and targeted therapies.

\section{Additional files}

Additional file 1: Supplemental Figures S1 - S14. Figure S1 - Time course approach to induce resistance to cetuximab and measure gene expression and DNA methylation changes; Figure S2 - Anchorageindependent growth of cetuximab generation 10 (CTX-G10); Figure S3 Heatmap and hierarchical clustering of gene expression values in 11 generations of SCC25 cells treated with PBS as control (black columns) and with $100 \mathrm{nM}$ of cetuximab (red columns) to acquire resistance.; Figure S4 - Time course gene expression comparison to previously known gene signatures of resistance to EGFR inhibitors; Figure S5 - Expected gene expression values for genes in each CoGAPS pattern inferred from gene expression data over generations of PBS control (black lines) or treatment with $100 \mathrm{nM}$ of cetuximab (red lines); Figure S6 - Heatmap of gene set analysis scores for targets of transcription factors in theEGFR network, targets of the AP-2alpha transcription factors associated with cetuximabresponse, and cetuximab resistance signatures in CoGAPS patterns; Figure S7 - Heatmaps of Pearson correlation coefficients between CoGAPS gene expression and DNA methylation patterns; Figure S8 - Gene expression heatmap for the time course experiment vs. single cell resistant clones experiment; Figure $\mathbf{S 9}$ - DNA methylation heatmap for the time course experiment vs. single cell resistant clones experiment; Figure S10 - Microscopy images of cetuximab single cell resistant clones (CTXR); Figure S11 - Epigenetically regulated pattern marker genes associated with resistance presenting significant anti-correlation between gene expression and DNA methylation in thecetuximab single cell resistant clones (CTXR); Figure S12 - Proliferation assay of cetuximab resistant single cell clones (CTXR); Figure S13 - Bar plot of FGFR1 expression in human tumor samples pre (black) and post (red) treatment with cetuximab; Figure S14 - Heatmap of FGF family genes methylation (A) and expression (B) in time coursedata for treated and control samples. (PDF $22593 \mathrm{~kb}$ )

Additional file 2: Table S1. PatternMarker genes for the expression and methylation CoGAPS patterns and list of genes with significant anticorrelation between gene expression and methylation. (XLSX $117 \mathrm{~kb}$ )

Additional file 3: Table S2. $P$ values of MSigDB hallmark pathways for CoGAPS expression patterns calculated with the permutation-based statistic in calcCoGAPSStat. (XLSX $99 \mathrm{~kb}$ )

Additional file 4: Table S3. $P$ values of MSigDB hallmark pathways for CoGAPS DNA methylation patterns calculated with the permutationbased statistic in calcCoGAPSStat. (XLSX $79 \mathrm{~kb}$ )

\footnotetext{
Abbreviations

AKT: AKT serine/threonine kinase; ATCC: American Type Culture Collection; CoGAPS: Coordinated Gene Activity in Pattern Sets; CTX: Cetuximab; CTXR: Single cell cetuximab resistant clone; DNA: Deoxyribonucleic acid; EGFR: Endogenous growth factor receptor; FDA: Food and Drug Administration; FGFR1: Fibroblast growth factor receptor 1; GEO: Gene Expression Omnibus; GSTP1: Glutathione S-Transferase pi 1: GWCoGAPS: Genome Wide Coordinated Gene Activity in Pattern Sets; HNSCC: Head and neck squamous cell carcinoma; HRAS: HRAS protooncogene; JHMI: Johns Hopkins Medical Institutions; LPFS: Long-progressionfree survival; MAPK: Mitogen activated kinase-like protein; NSCLC: Non-smallcell lung cancer; PBS: Phosphate buffered saline; PI3K: Phosphoinositide-3kinase regulatory subunit 1; RIN: RNA integrity number; RNA: Ribonucleic
}

acid; RNA-seq: Ribonucleic acid sequencing; rRNA: Ribosomal ribonucleic acid; SPFS: Short-progression-free survival; STR: Short tandem repeat; TCGA: The Cancer Genome Atlas; TFAP2A: Transcription factor AP-2 alpha

\section{Acknowledgements}

We thank JHMI Deep Sequencing \& Microarray Core and SKCCC Microarray Core Facility on performing and providing advice on RNA-seq and DNA methylation hybridization arrays, respectively; S. Boca, B. Kerr, S. Floor, C. Mak, T. Ou, D. Sidransky, L. M. Weiner, F. Zamuner, K. Zambo, and members of NewPISlack for critical comments and feedback during the preparation of the manuscript. In memory of Elizabeth Klein.

\section{Funding}

This work was supported by NIH Grants R01CA177669, R21DE025398, P30CA006973, R01DE017982, SPORE P50DE019032, and the Johns Hopkins University Catalyst Award.

\section{Availability of data and materials}

Unless otherwise specified, all genomics analyses were performed in $\mathrm{R}$ and code for these analyses is available from https://sourceforge.net/projects/ scc25timecourse. All transcriptional and epigenetic data of the cell lines from this paper have been deposited in GEO (GSE98815). DNA methylation data of head and neck tumors after treatment with cetuximab are available in GEO (GSE110996).

\section{Authors' contributions}

GS, LTK, SL, CHC, and EJF planned, designed and wrote the manuscript with input from all authors. GS, LTK, SL, MT, CHC, and EJF contributed to the development of methodology. SS provided DNA from HNSCC patients following cetuximab treatment for DNA methylation analysis. GS, SL, and EJF performed analysis and interpretation of data (e.g. computational analysis). $R R, H O, H C, M C, A F, L V D, S S, J A$, and DAG participated in development of methodology and provided technical and material support. RR, LVD, El, and DAG participated in the review and/or revision of the manuscript. All authors discussed the data and contributed to the manuscript preparation. $\mathrm{CHC}$ and EJF instigated and supervised the project. All authors read and approved the final manuscript.

\section{Ethics approval and consent to participate}

HNSCC samples and patient information collection were approved by the Independent Ethics Committee and the Belgian Health Authorities (ID: 2008/ 20MAR/090) and were conducted in accordance with the Declaration of Helsinki (October 2000). It was prospectively planned to perform translational research and patients gave their informed consent for repeated biopsies.

\section{Competing interests}

The authors declare that they have no competing interests.

\section{Publisher's Note}

Springer Nature remains neutral with regard to jurisdictional claims in published maps and institutional affiliations.

\section{Author details}

${ }^{1}$ Institute of Genetic Medicine, Johns Hopkins University, Baltimore, MD, USA. ${ }^{2}$ Department of Oncology, Sidney Kimmel Comprehensive Cancer Center, Johns Hopkins University, Baltimore, MD, USA. ${ }^{3}$ Department of Head and Neck-Endocrine Oncology, Moffitt Cancer Center, Tampa, FL, USA. ${ }^{4}$ Department of Otorhinolaryngology-Head and Neck Surgery, Keio University School of Medicine, Tokyo, Japan. ${ }^{5}$ Department of Surgery - OtolaryngologyHead and Neck Surgery, University of Utah, |Salt Lake City, UT, USA. ${ }^{6} \mathrm{Head}$ and Neck Surgery Unit, St Luc University Hospital, Brussels, Belgium.

${ }^{7}$ Laboratory of Systems Biology and Computational Genetics, Vavilov Institute of General Genetics, Russian Academy of Sciences, Moscow, Russia. ${ }^{8}$ Department of Surgery, UC San Diego Moores Cancer Center, La Jolla, CA, USA. ${ }^{9}$ Department of Otolaryngology-Head and Neck Surgery, Johns Hopkins University, Baltimore, MD, USA. 


\section{Received: 23 January 2018 Accepted: 1 May 2018}

\section{Published online: 23 May 2018}

\section{References}

1. Sawyers C. Targeted cancer therapy. Nature. 2004;432:294-7.

2. Hyman DM, Taylor BS, Baselga J. Implementing Genome-Driven Oncology. Cell. 2017:168:584-99.

3. Engelman JA, Zejnullahu K, Mitsudomi T, Song Y, Hyland C, Park JO, et al. MET amplification leads to gefitinib resistance in lung cancer by activating ERBB3 signaling. Science. 2007;316:1039-43.

4. Hata AN, Niederst MJ, Archibald HL, Gomez-Caraballo M, Siddiqui FM, Mulvey $\mathrm{HE}$, et al. Tumor cells can follow distinct evolutionary paths to become resistant to epidermal growth factor receptor inhibition. Nat Med. 2016;22:262-9

5. Misale S, Yaeger R, Hobor S, Scala E, Janakiraman M, Liska D, et al. Emergence of KRAS mutations and acquired resistance to anti-EGFR therapy in colorectal cancer. Nature. 2012;486:532-6.

6. Pietrantonio F, Vernieri C, Siravegna G, Mennitto A, Berenato R, Perrone F, et al. Heterogeneity of acquired resistance to anti-EGFR monoclonal antibodies in patients with metastatic colorectal cancer. Clin Cancer Res. 2017;23:2414-22.

7. Vincenzi B, Zoccoli A, Pantano F, Venditti O, Galluzzo S. Cetuximab: from bench to bedside. Curr Cancer Drug Targets. 2010;10:80-95.

8. Boeckx C, Weyn C, Vanden Bempt I, Deschoolmeester V, Wouters A, Specenier $\mathrm{P}$, et al. Mutation analysis of genes in the EGFR pathway in Head and Neck cancer patients: implications for anti-EGFR treatment response. BMC Res Notes. 2014;7:337.

9. Quesnelle KM, Wheeler SE, Ratay MK, Grandis JR. Preclinical modeling of EGFR inhibitor resistance in head and neck cancer. Cancer Biol Ther. 2012; 13:935-45.

10. Wheeler DL, Huang S, Kruser TJ, Nechrebecki MM, Armstrong EA, Benavente S, et al. Mechanisms of acquired resistance to cetuximab: role of HER (ErbB) family members. Oncogene. 2008;27:3944-56.

11. Narayan M, Wilken JA, Harris LN, Baron AT, Kimbler KD, Maihle NJ. Trastuzumab-induced HER reprogramming in "resistant" breast carcinoma cells. Cancer Res. 2009;69:2191-4.

12. Smyth GK. Linear models and empirical Bayes methods for assessing differential expression in microarray experiments. Stat Appl Genet Mol Biol. 2004;3:Article3.

13. Ernst J, Nau GJ, Bar-Joseph Z. Clustering short time series gene expression data. Bioinforma Oxf Engl. 2005;21(Suppl 1):1159-68.

14. Lin D, Shkedy Z, Yekutieli D, Burzykowski T, Göhlmann HWH, De Bondt A, et al. Testing for trends in dose-response microarray experiments: a comparison of several testing procedures, multiplicity and resampling-based inference. Stat Appl Genet Mol Biol. 2007;6:Article26.

15. Aryee MJ, Gutiérrez-Pabello JA, Kramnik I, Maiti T, Quackenbush J. An improved empirical bayes approach to estimating differential gene expression in microarray time-course data: BETR (Bayesian Estimation of Temporal Regulation). BMC Bioinformatics. 2009;10:409.

16. Liao JC, Boscolo R, Yang Y-L, Tran LM, Sabatti C, Roychowdhury VP. Network component analysis: reconstruction of regulatory signals in biological systems. Proc Natl Acad Sci U S A. 2003;100:15522-7.

17. Bonneau R, Reiss DJ, Shannon P, Facciotti M, Hood L, Baliga NS, et al. The Inferelator: an algorithm for learning parsimonious regulatory networks from systems-biology data sets de novo. Genome Biol. 2006;7:R36.

18. Ernst J, Vainas O, Harbison CT, Simon I, Bar-Joseph Z. Reconstructing dynamic regulatory maps. Mol Syst Biol. 2007;3:74.

19. Seok J, Xiao W, Moldawer LL, Davis RW, Covert MW. A dynamic network of transcription in LPS-treated human subjects. BMC Syst Biol. 2009;3:78.

20. Naegle KM, Welsch RE, Yaffe MB, White FM, Lauffenburger DA. MCAM: multiple clustering analysis methodology for deriving hypotheses and insights from high-throughput proteomic datasets. PLoS Comput Biol. 2011;7:e1002119.

21. Fernández MA, Rueda C, Peddada SD. Identification of a core set of signature cell cycle genes whose relative order of time to peak expression is conserved across species. Nucleic Acids Res. 2012;40:2823-32.

22. Wise A, Bar-Joseph Z. SMARTS: reconstructing disease response networks from multiple individuals using time series gene expression data. Bioinforma Oxf Engl. 2015:31:1250-7.

23. Fertig EJ, Ding J, Favorov AV, Parmigiani G, Ochs MF. CoGAPS: an R/C++ package to identify patterns and biological process activity in transcriptomic data. Bioinforma Oxf Engl. 2010;26:2792-3.
24. Ochs MF, Fertig EJ. Matrix factorization for transcriptional regulatory network inference. IEEE Symp Comput Intell Bioinforma Comput Biol Proc. 2012;2012:387-96

25. Fertig EJ, Markovic A, Danilova LV, Gaykalova DA, Cope L, Chung CH, et al. Preferential activation of the hedgehog pathway by epigenetic modulations in HPV negative HNSCC identified with meta-pathway analysis. PLOS One. 2013; e78127:8.

26. Stein-O'Brien GL, Carey JL, Lee WS, Considine M, Favorov AV, Flam E, et al. PatternMarkers \& GWCoGAPS for novel data-driven biomarkers via whole transcriptome NMF. Bioinformatics. 2017;33:1892-4.

27. Azuma K, Kawahara A, Sonoda K, Nakashima K, Tashiro K, Watari K, et al. FGFR1 activation is an escape mechanism in human lung cancer cells resistant to afatinib, a pan-EGFR family kinase inhibitor. Oncotarget. 2014;5:5908-19.

28. Bertotti A, Papp E, Jones S, Adleff V, Anagnostou V, Lupo B, et al. The genomic landscape of response to EGFR blockade in colorectal cancer. Nature. 2015;526:263-7.

29. Koole K, Brunen D, van Kempen PMW, Noorlag R, de Bree $R$, Lieftink $C$, et al. FGFR1 is a potential prognostic biomarker and therapeutic target in head and neck squamous cell carcinoma. Clin Cancer Res. 2016:22:3884-93.

30. Hatakeyama $H$, Cheng $H$, Wirth $P$, Counsell $A$, Marcrom SR, Wood CB, et al. Regulation of heparin-binding EGF-like growth factor by miR-212 and acquired cetuximab-resistance in head and neck squamous cell carcinoma. PLoS One. 2010;e12702:5.

31. Cheng H, Fertig EJ, Ozawa $H$, Hatakeyama H, Howard JD, Perez J, et al. Decreased SMAD4 expression is associated with induction of epithelial-tomesenchymal transition and cetuximab resistance in head and neck squamous cell carcinoma. Cancer Biol Ther. 2015;16:1252-8.

32. Wang K, Singh D, Zeng Z, Coleman SJ, Huang Y, Savich GL, et al. Mapsplice: Accurate mapping of RNA-seq reads for splice junction discovery. Nucleic Acids Res. 2010;38:e178.

33. Li B, Dewey CN. RSEM: accurate transcript quantification from RNA-Seq data with or without a reference genome. BMC Bioinformatics. 2011;12:323.

34. Fortin J-P, Labbe A, Lemire M, Zanke BW, Hudson TJ, Fertig EJ, et al. Functional normalization of 450k methylation array data improves replication in large cancer studies. Genome Biol. 2014;15:503.

35. Bidaut G. Interpreting and comparing clustering experiments through graph visualization and ontology statistical enrichment with the ClutrFree package. In: Ochs MF, Casagrande JT, Davuluri RV, editors. Biomed. Inform. Cancer Res. Boston, MA: Springer US; 2010. p. 315-33. http://link.springer.com/10. 1007/978-1-4419-5714-6_19. Accessed 23 Jan 2018.

36. Lawrence MS, Sougnez C, Lichtenstein L, Cibulskis K, Lander E, Gabriel SB, et al. Comprehensive genomic characterization of head and neck squamous cell carcinomas. Nature. 2015;517:576-82.

37. Subramanian A, Tamayo P, Mootha VK, Mukherjee S, Ebert BL, Gillette MA et al. Gene set enrichment analysis: a knowledge-based approach for interpreting genome-wide expression profiles. Proc Natl Acad Sci U S A. 2005;102:15545-50.

38. Coldren CD. Baseline gene expression predicts sensitivity to gefitinib in non-small cell lung cancer cell lines. Mol Cancer Res. 2006;4:521-8.

39. Fertig EJ, Ren Q, Cheng H, Hatakeyama H, Dicker AP, Rodeck U, et al. Gene expression signatures modulated by epidermal growth factor receptor activation and their relationship to cetuximab resistance in head and neck squamous cell carcinoma. BMC Genomics. 2012;13:160.

40. McCall MN, Bolstad BM, Irizarry RA. Frozen robust multiarray analysis (fRMA). Biostat Oxf Engl. 2010;11:242-53.

41. Fertig EJ, Ozawa H, Thakar M, Howard JD, Kagohara LT, Krigsfeld G, et al. CoGAPS matrix factorization algorithm identifies transcriptional changes in AP-2alpha target genes in feedback from therapeutic inhibition of the EGFR network. Oncotarget. 2016;7:73845-64.

42. Bossi P, Bergamini C, Siano M, Cossu Rocca M, Sponghini AP, Favales F, et al. Functional genomics uncover the biology behind the responsiveness of head and neck squamous cell cancer patients to cetuximab. Clin Cancer Res. 2016:22:3961-70.

43. Schmitz S, Bindea G, Albu RI, Mlecnik B, Machiels J-P. Cetuximab promotes epithelial to mesenchymal transition and cancer associated fibroblasts in patients with head and neck cancer. Oncotarget. 2015:6:34288-99

44. Fortin J-P, Triche TJ, Hansen KD. Preprocessing, normalization and integration of the Illumina HumanMethylationEPIC array with minfi. Bioinformatics. 2017;33:558-60. 
45. Mootha VK, Lindgren CM, Eriksson K-F, Subramanian A, Sihag S, Lehar J, et al. PGC-1a-responsive genes involved in oxidative phosphorylation are coordinately downregulated in human diabetes. Nat Genet. 2003; 34:267-73.

46. Marek L, Ware KE, Fritzsche A, Hercule P, Helton WR, Smith JE, et al. Fibroblast growth factor (FGF) and FGF receptor-mediated autocrine signaling in nonsmall-cell lung cancer cells. Mol Pharmacol. 2009;75:196-207.

47. Marshall ME, Hinz TK, Kono SA, Singleton KR, Bichon B, Ware KE, et al. Fibroblast growth factor receptors are components of autocrine signaling networks in head and neck squamous cell carcinoma cells. Clin Cancer Res. 2011;17:5016-25.

48. Wynes MW, Hinz TK, Gao D, Martini M, Marek LA, Ware KE, et al. FGFR1 mRNA and protein expression, not gene copy number, predict FGFR TKI sensitivity across all lung cancer histologies. Clin Cancer Res. 2014;20:3299-309.

49. Ochs MF, Rink L, Tarn C, Mburu S, Taguchi T, Eisenberg B, et al. Detection of treatment-induced changes in signaling pathways in gastrointestinal stromal tumors using transcriptomic data. Cancer Res. 2009;69:9125-32.

50. Hafner M, Niepel M, Chung M, Sorger PK. Growth rate inhibition metrics correct for confounders in measuring sensitivity to cancer drugs. Nat Methods. 2016;13:521-7.

51. Hill SM, Heiser LM, Cokelaer T, Unger M, Nesser NK, Carlin DE, et al. Inferring causal molecular networks: empirical assessment through a communitybased effort. Nat Methods. 2016;13:310-8.

52. Zhu X, Ching T, Pan X, Weissman SM, Garmire L. Detecting heterogeneity in singlecell RNA-Seq data by non-negative matrix factorization. PeerJ. 2017;5:e2888.

53. Tyekucheva S, Marchionni L, Karchin R, Parmigiani G. Integrating diverse genomic data using gene sets. Genome Biol. 2011;12:R105.

54. Bachman KE, Park BH, Rhee I, Rajagopalan H, Herman JG, Baylin SB, et al. Histone modifications and silencing prior to DNA methylation of a tumor suppressor gene. Cancer Cell. 2003;3:89-95.

55. Stirzaker C, Song JZ, Davidson B, Clark SJ. Transcriptional gene silencing promotes DNA hypermethylation through a sequential change in chromatin modifications in cancer cells. Cancer Res. 2004;64:3871-7.

56. Shaffer SM, Dunagin MC, Torborg SR, Torre EA, Emert B, Krepler C, et al. Rare cell variability and drug-induced reprogramming as a mode of cancer drug resistance. Nature. 2017;546:431-5.

57. Babina IS, Turner NC. Advances and challenges in targeting FGFR signalling in cancer. Nat Rev Cancer 2017;17:318-32.

58. Goldstein M, Meller I, Orr-Urtreger A. FGFR1 over-expression in primary rhabdomyosarcoma tumors is associated with hypomethylation of a $5^{\prime} \mathrm{CpG}$ island and abnormal expression of the AKT1, NOG, and BMP4 genes. Genes Chromosomes Cancer. 2007:46:1028-38.

59. Rand V, Huang J, Stockwell T, Ferriera S, Buzko O, Levy S, et al. Sequence survey of receptor tyrosine kinases reveals mutations in glioblastomas. Proc Natl Acad Sci U S A. 2005;102:14344-9.

60. Andre F, Bachelot T, Campone M, Dalenc F, Perez-Garcia JM, Hurvitz SA, et al. Targeting FGFR with Dovitinib (TKI258): preclinical and clinical data in breast cancer. Clin Cancer Res. 2013;19:3693-702.

61. Cihoric N, Savic S, Schneider S, Ackermann I, Bichsel-Naef M, Schmid RA, et al. Prognostic role of FGFR1 amplification in early-stage non-small cell lung cancer. Br J Cancer. 2014;110:2914-22.

62. Armstrong K, Ahmad I, Kalna G, Tan SS, Edwards J, Robson CN, et al. Upregulated FGFR1 expression is associated with the transition of hormonenaive to castrate-resistant prostate cancer. Br J Cancer. 2011;105:1362-9.

63. Tomlinson DC, Lamont FR, Shnyder SD, Knowles MA. Fibroblast growth factor receptor 1 promotes proliferation and survival via activation of the mitogen-activated protein kinase pathway in bladder cancer. Cancer Res. 2009;69:4613-20

64. Gorringe KL, Jacobs S, Thompson ER, Sridhar A, Qiu W, Choong DYH, et al. High-resolution single nucleotide polymorphism array analysis of epithelial ovarian cancer reveals numerous microdeletions and amplifications. Clin Cancer Res. 2007;13:4731-9.

65. Göke F, Bode M, Franzen A, Kirsten R, Goltz D, Göke A, et al. Fibroblast growth factor receptor 1 amplification is a common event in squamous cell carcinoma of the head and neck. Mod Pathol. 2013;26:1298-306.

66. Clauditz TS, Böttcher A, Hanken H, Borgmann K, Sauter G, Wilczak W, et al. Prevalence of fibroblast growth factor receptor 1 (FGFR1) amplification in squamous cell carcinomas of the head and neck. J Cancer Res Clin Oncol. 2018;144:53-61.
67. Puram SV, Tirosh I, Parikh AS, Patel AP, Yizhak K, Gillespie S, et al. Single-cell transcriptomic analysis of primary and metastatic tumor ecosystems in head and neck cancer. Cell. 2017;171:1611-1624.e24.

68. Niehr F, Eder T, Pilz T, Konschak R, Treue D, Klauschen F, et al. Multilayered omics-based analysis of a head and neck cancer model of cisplatin resistance reveals intratumoral heterogeneity and treatment-induced clonal selection. Clin Cancer Res. 2018;24:158-68.

\section{Ready to submit your research? Choose BMC and benefit from:}

- fast, convenient online submission

- thorough peer review by experienced researchers in your field

- rapid publication on acceptance

- support for research data, including large and complex data types

- gold Open Access which fosters wider collaboration and increased citations

- maximum visibility for your research: over $100 \mathrm{M}$ website views per year

At BMC, research is always in progress.

Learn more biomedcentral.com/submissions 